\title{
Lipid accumulation of Chlorella sp. TLD6B from the Taklimakan Desert under salt stress
}

\author{
Hong $\mathrm{Li}^{1}{ }^{1}$, Jun $\operatorname{Tan}^{2}$, Yun Mu ${ }^{2}$, Jianfeng Gao ${ }^{\text {Corresp. } 2}$ \\ ${ }^{1}$ College of Life Sciences; Key Laboratory of Xinjiang Phytomedicine Resource and Utilization, Ministry of Education, School of Pharmacy, Shihezi \\ University, Shihezi, Xinjiang, China \\ ${ }^{2}$ College of Life Sciences, Shihezi University, Shihezi, Xinjiang, China \\ Corresponding Author: Jianfeng Gao \\ Email address: jianfengg@shzu.edu.cn
}

Chlorella has become an important raw material for biodiesel production in recent years, and Chlorella sp. TLD6B, a species with high lipid concentrations and high salt and drought tolerance, has been cultivated on a large scale. To explore the lipid accumulation of Chlorella sp. TLD6B and its relationship to external $\mathrm{NaCl}$ concentrations, we performed physiological measurements and genome-wide gene expression profiling under different levels of salt stress. Chlorella sp. TLD6B was able to tolerate high levels of salt stress (0.8 $\mathrm{M} \mathrm{NaCl}$ addition). Lipid concentrations initially increased and then decreased as salt stress increased and were highest under the addition of $0.2 \mathrm{M} \mathrm{NaCl}$. Comparative transcriptomic analysis revealed that salt stress enhanced the expression of genes related to sugar metabolism and fatty acid biosynthesis (the ACCases BC and BCCP, KAS II, and GPDHs involved in TAG synthesis), thereby promoting lipid accumulation under the addition of 0.2 $\mathrm{M} \mathrm{NaCl}$. However, high salinity inhibited cell growth. Expression of three SADs, whose encoded products function in unsaturated fatty acid biosynthesis, was up-regulated under high salinity (0.8 M NaCl addition). This research clarifies the relationship between salt tolerance and lipid accumulation and promotes the utilization of Chlorella sp. TLD6B. 
1 Lipid accumulation of Chlorella sp. TLD6B from the Taklimakan Desert 2 under salt stress

3 Hong $\mathrm{Li}^{1,2}$, Jun $\mathrm{Tan}^{1}$, Yun $\mathrm{Mu}^{1}$, Jianfeng $\mathrm{Gao}^{1, *}$

$4 \quad{ }^{1}$ College of Life Sciences, Shihezi University, Shihezi, Xinjiang, China

$5 \quad{ }^{2}$ Key Laboratory of Xinjiang Phytomedicine Resource and Utilization, Ministry of Education, School of

6 Pharmacy, Shihezi University, Shehezi, Xinjiang 832003, China

$7 \quad{ }^{*}$ Corresponding author.

9 Corresponding Author:

10 Jianfeng $\mathrm{Gao}^{1}$

11 Beisi Road, Shihezi, Xinjiang, 832000, China

12 Email address: jianfengg@shzu.edu.cn

\section{Abstract}

Chlorella has become an important raw material for biodiesel production in recent years, and Chlorella sp. TLD6B, a species with high lipid concentrations and high salt and drought tolerance, has been cultivated on a large scale. To explore the lipid accumulation of Chlorella $\mathrm{sp}$. TLD6B and its relationship to external $\mathrm{NaCl}$ concentrations, we performed physiological measurements and genome-wide gene expression profiling under different levels of salt stress. Chlorella sp. TLD6B was able to tolerate high levels of salt stress ( $0.8 \mathrm{M} \mathrm{NaCl}$ addition). Lipid concentrations initially increased and then decreased as salt stress increased and were highest under the addition of $0.2 \mathrm{M} \mathrm{NaCl}$. Comparative transcriptomic analysis revealed that salt stress enhanced the expression of genes related to sugar metabolism and fatty acid biosynthesis (the $A C C a s e s B C$ and BCCP, KAS II, and GPDHs involved in TAG synthesis), thereby promoting lipid accumulation under the addition of $0.2 \mathrm{M} \mathrm{NaCl}$. However, high salinity inhibited cell growth. Expression of three $S A D s$, whose encoded products function in unsaturated fatty acid biosynthesis, was up-regulated under high salinity $(0.8 \mathrm{M} \mathrm{NaCl}$ addition). This research clarifies the relationship between salt tolerance and lipid accumulation and promotes the utilization of Chlorella sp. TLD6B.

\section{Subjects:Agricultural and Biological Sciences, Microbiology, Plant Science}

Keywords: Salt stress, Chlorella sp. TLD6B, Transcriptome, Lipid accumulation

\section{INTRODUCTION}

Increasing global energy demands cause over-exploitation and shortages of nonrenewable resources such as oil, natural gas, and coal and increase the urgency associated with research on bioenergy. As photoautotrophic organisms (Schenk et al., 2008), microalgae are characterized by high lipid concentrations and rapid growth, which makes them the most promising organisms for the development of biodiesel (Halim et al., 2012). The lipid metabolism of microalgae is a 
complex process that involves the synthesis and metabolism of fatty acids, triglycerides (TAGs), acetyl-CoA, and other compounds, and it is closely related to algal growth and stress tolerance (Gao et al., 2014). Environmental changes alter the expression of many genes encoding enzymes in these metabolic pathways and thereby influence microalgal lipid metabolism. Previous studies have found that lipid accumulation in microalgae is affected by changes in the expression of the ACCase subunit genes $B C, B C C P$, and $\alpha-C T$, the fatty acid synthesis genes $S A D, K A S I I, M C A T$, as well as the TAG synthesis genes GPAT, PAP, LPATT, and DGAT (Zalutskaya et al., 2015; Huang et al., 2016). At present, the detailed signaling mechanisms that regulate lipid metabolism in microalgae remain unclear. However, lipid accumulation is known to be regulated by abscisic acid (ABA) and reactive oxygen species (ROS) signals (Gao et al., 2014; Wang et al., 2016), indicating that microalgal lipid metabolism is closely related to adaptation to unfavorable environments.

Chlorella is a eukaryotic, single-celled green microalga that is widely distributed in fresh water, soils, and deserts ( $L i$ et al., 2016). It has been widely used in research related to microalgae energy production (Liang et al., 2009; Li et al., 2011; Liu et al., 2011). Researchers currently focus primarily on freshwater Chlorella, and there have been few studies of desert Chlorella. Desert Chlorella grows in an extremely arid environment, with a maximum temperature of $67.2^{\circ} \mathrm{C}$ in intense heat and a temperature difference of more than $40{ }^{\circ} \mathrm{C}$ between day and night. The winter is cold, with low precipitation and high evaporation (Zhang et al., 2020). Lewis and Flechtner (2002) found that desert green algae evolved from aquatic green algae at least five independent times. Therefore, desert Chlorella has adapted to the extremely harsh arid environment, making it an excellent material for studying the molecular biology of stress resistance at the single-cell level (Mu et al., 2016). In the past, studies of desert algae have focused mainly on the algal composition of microbiotic crusts and the characteristics and functions of crust formation (Ashley \& Rushforth, 1984; Garcia-Pichel et al., 2001; Lewis \& Flechtner, 2002; Zhao et al., 2006). In recent years, Li et al. (2012) and Wang et al. (2014) analyzed the phylogeny of several strains of microalgae in the Gurbantonggut and Taklimakan Deserts. Among these algae, GTD7c-2, TLD2A, TLD6B are classified as Chlorella. Gong et al. (2013) compared different cultivation methods for desert Chlorella GTD8A1. Zhu et al. (2014) and Mu et al. (2016) measured the oil content of desert microalgae and desert Chlorella, respectively, and made a preliminary assessment of their potential for conversion to biodiesel.

Previous studies on lipid accumulation in freshwater Chlorella under salt stress have provided useful information, but there have been no reports on the mechanisms of lipid accumulation in desert Chlorella under salt stress. Desert Chlorella sp. TLD6B differs from fresh water algae, as it grows in extremely harsh environments. We must first clarify the mechanistic relationship between salinity stress and lipid accumulation before we can further develop desert Chlorella sp. TLD6B for use in biofuel production. Studies have shown that lipid accumulation in Chlorella can be induced by salt stress (Wang et al., 2016) and that salinity enhances microalgal lipid accumulation for biofuel production (BenMoussa-Dahmen et al., 2016; Srivastava et al., 2017). High salinity is an important factor that stimulates lipid 
accumulation, but it also has adverse effects on biomass growth and lipid production in microalgae (Markou \& Nerantzis, 2013; Ho et al., 2014). Sharma and Chauhan (2016) used comparative transcriptomics to identify the molecular components associated with differences in lipid accumulation between the microalgal species Scenedesmus dimorphus and S. quadricauda. Gao et al. (2014) used transcriptomics, genomics, and proteomics to characterize lipid accumulation mechanisms of the oleaginous microalga $C$. protothecoides.

Here, we studied Chlorella sp. TLD6B, a desert Chlorella collected from the Taklimakan Desert that exhibits greater drought and salt tolerance than freshwater Chlorella (unpublished results). We investigated the mechanism of lipid accumulation in Chlorella sp. TLD6B under salt stress by measuring relevant physiological parameters and analyzing whole-genome gene expression profiles under different levels of salt stress. This research aims to clarify the relationship between salt stress and oil accumulation in Chlorella sp. TLD6B and to promote its development and utilization in biofuel production.

\section{MATERIALS AND METHODS}

\section{Algal strain and culture conditions}

The experimental algae were collected from the Taklimakan Desert $\left(37^{\circ} 36^{\prime} \mathrm{N}, 80^{\circ} 23^{\prime} \mathrm{E}, 1254 \mathrm{~m}\right.$ altitude, $3.5^{\circ} \mathrm{C}$ average surface temperature, $0.4 \mathrm{M}$ average $\mathrm{NaCl}$ salt concentration, $71.2 \mathrm{~W} \cdot \mathrm{m}^{-2}$ net radiation at 16:00) in Xinjiang Province, China. Field experiments were approved by the College of Life Sciences of Shihezi University (project number: 2011-GB-200900-G). The algae were isolated in the laboratory from a mixed culture of Taklimakan Desert soil samples and were identified as Chlorella sp. TLD6B (Wang et al., 2014). They were maintained in an autotrophic culture system at the College of Life Sciences, Shihezi University, China. Bold basal medium (BBM; Nichols, 1973) was autoclaved, and algal cells in the logarithmic growth phase were inoculated into Erlenmeyer flasks containing $500 \mathrm{~mL}$ BBM medium at a ratio of $20 \%(\mathrm{~V} / \mathrm{V})$. The initial cell density was controlled at an $\mathrm{OD}_{680}$ of approximately 0.1 , and the preparation of BBM medium is described in Table S1. Light was provided by white fluorescent lamps at an intensity of $72 \mu \mathrm{mol}$ photons $\mathrm{m}^{-2} \mathrm{~s}^{-1}$ with a $12 \mathrm{~h} / 12 \mathrm{~h}$ light-dark cycle, and the temperature was $23^{\circ} \mathrm{C}$. Flasks were shaken three times a day at regular intervals and cultured for $24 \mathrm{~d}$ (Mu et al., 2016).

\section{Salt stress treatments}

Algal cells in the logarithmic growth phase were inoculated into Erlenmeyer flasks containing $300 \mathrm{~mL}$ BBM medium, and $0.0,0.1,0.2,0.4,0.6$, or $0.8 \mathrm{M} \mathrm{NaCl}$ was added to supplement the medium to a final volume of $500 \mathrm{~mL}$. The $0.0 \mathrm{NaCl}$ treatment was the control, and there were three replicates of each treatment. Algal culture was performed as described above. Samples were obtained every $6 \mathrm{~d}$ after inoculation to measure physiological and biochemical indexes such as biomass, carbohydrate concentration, and total lipid concentration.

\section{Measurement of physiological and biochemical indexes}

Culture solution $(100 \mathrm{~mL})$ was added to $10-\mathrm{mL}$ centrifuge tubes at $4{ }^{\circ} \mathrm{C}$, and the tubes were 
116

117

118

119

120

121

122

123

124

125

126

127

128

129

130

131

132

133

134

135

136

137

138

139

140

141

142

143

144

145

146

147

148

149

150

151

152

153

52

3

centrifuged at $8000 \mathrm{rpm}$ for $15 \mathrm{~min}$. The supernatant was discarded, and $2 \mathrm{~mL}$ of deionized water was added. The cell sediments were washed three times by vortexing (5-10 s each time), then divided into two equal parts. One part was used for dry weight measurement, and the other was immediately frozen in liquid nitrogen and stored at $-80^{\circ} \mathrm{C}$ for further analysis.

Optical density at $680 \mathrm{~nm}\left(\mathrm{OD}_{680}\right)$ was determined using a UV-5800H UV-VIS spectrophotometer (Metash, Shanghai, China), and a growth curve was drawn (Gong et al., 2013). The centrifuged Chlorella sp. TLD6B sediment was dried at $80{ }^{\circ} \mathrm{C}$ for $24 \mathrm{~h}$ to measure the dry weight (Wan et al., 2015). The specific growth rate was calculated using the method of Wang et al. (2016).

$$
\mu=\frac{\operatorname{lgN}_{1}-\operatorname{lgN}_{0}}{\mathrm{t}_{1}-\mathrm{t}_{0}} \times 3.322
$$

Where $\mu$ is the specific growth rate, $\mathrm{mg} \mathrm{L}^{-1} \mathrm{~d}^{-1} ; \mathrm{N}_{0}$ is the biomass from the previous sampling date, $\mathrm{mg} \mathrm{L}^{-1} ; \mathrm{N}_{1}$ is the current biomass, $\mathrm{mg} \mathrm{L}^{-1}$; and $\mathrm{t}_{0}$ and $\mathrm{t}_{1}$ are the culture times, $\mathrm{t}_{1}-\mathrm{t}_{0}$ $=6 \mathrm{~d}$.

The phenol-sulfuric acid method was used to measure carbohydrate content (Dubois et al., 1956). The absorbance was measured at $490 \mathrm{~nm}$ on an iMark Microplate Reader (BIO-RAD, USA). Different concentrations of glucose were used to create a standard curve $(y=8.55 x-0.07$, where $\mathrm{x}$ is the standard concentration $\left[\mu \mathrm{g} \mathrm{mL}^{-1}\right]$ and $\mathrm{y}$ is the absorbance value), from which the glucose concentration $\mathrm{X}_{\mathrm{C}}$ of the sample could be calculated. $\mathrm{X}_{\mathrm{C}}\left(\mu \mathrm{g} \mathrm{mL} \mathrm{m}^{-1}\right)$ was defined as shown in equation (Eq.) (2), and carbohydrate content (CC, \%) was defined as shown in Eq. (3).

$$
\begin{aligned}
& \mathrm{X}_{\mathrm{C}}=\frac{(\Delta \mathrm{A}+0.07)}{8.55} \\
& \mathrm{CC}=\frac{\mathrm{X}_{\mathrm{C}} \times \mathrm{V} \times 100 \%}{\mathrm{~V}_{\mathrm{C}} \times \mathrm{W}}
\end{aligned}
$$

where $\Delta \mathrm{A}$ is the absorbance at $490 \mathrm{~nm}, \mathrm{~V}$ is the total volume of the sample $(\mathrm{mL}), \mathrm{V}_{\mathrm{C}}$ is the volume of the subsample used for measurement $(\mu \mathrm{L})$, and $\mathrm{W}$ is the dry weight of the sample $(\mathrm{g}$ $\left.\mathrm{L}^{-1}\right)$.

The lipid content was measured by the colorimetric sulfo-phospho-vanillin method as described by Mishra et al. (2014) with some modifications (Li et al., 2012). The absorbance was measured at $528 \mathrm{~nm}$, and the corresponding linoleic acid concentration $\left(\mu \mathrm{g} \mathrm{mL}^{-1}\right)$ was calculated from the standard curve $(\mathrm{y}=0.0005 \mathrm{x}+0.008) . \mathrm{X}_{\mathrm{L}}\left(\mu \mathrm{g} \mathrm{mL} \mathrm{L}^{-1}\right)$ was defined as shown in Eq. (4), and the lipid content (LC, \%) was defined as shown in Eq. (5).

$$
\mathrm{X}_{\mathrm{L}}=\frac{(\Delta \mathrm{A}-0.008)}{0.0005}
$$

$$
\mathrm{LC}=\frac{\mathrm{X}_{\mathrm{L}} \times \mathrm{V} \times 100 \%}{\mathrm{~V}_{\mathrm{L}} \times \mathrm{W}}
$$


154

155

156

157

158

159

160

161

162

163

164

165

166

167

168

169

170

171

172

173

174

175

176

177

178

179

180

181

182

183

184

185

186

187

188

189

190

191

192

where $\Delta \mathrm{A}$ is the absorbance at $528 \mathrm{~nm}, \mathrm{~V}$ is the total volume of the extract $(\mathrm{mL}), \mathrm{V}_{\mathrm{L}}$ is the volume of the subsample used for measurement $(\mu \mathrm{L})$, and $\mathrm{W}$ is the dry weight of the sample $(\mathrm{g}$ $\left.\mathrm{L}^{-1}\right)$.

\section{RNA extraction and sequencing}

Based on the results of physiological measurements under salt stress, we selected Chlorella sp. TLD6B cells grown under low salt stress $(0.1 \mathrm{M} \mathrm{NaCl}$ addition $)$ and high salt stress $(0.8 \mathrm{M} \mathrm{NaCl}$ addition) for $18 \mathrm{~d}$ for use in transcriptome sequencing and analysis. Samples collected from the control group, the $0.1 \mathrm{M} \mathrm{NaCl}$ treatment group, and the $0.8 \mathrm{M} \mathrm{NaCl}$ treatment group were labeled CK18, Nacl1, and Nacl2, respectively. Each treatment was replicated two times. Samples were centrifuged at $8000 \mathrm{rpm}$ for $8 \mathrm{~min}$ at $4{ }^{\circ} \mathrm{C}$. After removal of the supernatants, Chlorella sp. TLD6B cells were washed with distilled water three times and stored at $-80{ }^{\circ} \mathrm{C}$ for RNA extraction.

Total RNA was isolated from all samples using the TRIzol reagent (Invitrogen, USA) according to the manufacturer's instructions. Total RNA was sent to Beijing Novogene Technology Co., Ltd. for RNA quality assessment, library construction, and sequencing (150-bp paired-end reads) on the Illumina NovaSeq 6000 platform. Raw sequence reads have been deposited into the NCBI GEO under accession number GSE162916.

\section{Transcriptome analysis}

The total raw reads were processed using FASTQC software with default parameters. Highquality clean reads were obtained by removing reads that contained adapters, reads that contained more than $10 \%$ unknown nucleotides $(\mathrm{N})$, and low-quality reads that contained more than $50 \%$ low-quality bases $\left(\mathrm{Q}_{\text {phred }} \leq 20\right)$. At the same time, the GC content, Q20, Q30, and sequence repetition level of the clean reads were calculated. Transcriptome de novo assembly was performed using Trinity v2.4.0 software (Grabherr et al., 2011) with the parameter Kmer $=25$. Corset v1.05 (Davidson \& Oshlack, 2014) was used with default parameters to cluster the transcript sequences in order to remove redundant sequences and obtain unigene sequence sets for subsequent analysis. The longest transcript of each subcomponent was used as the unigene for functional annotation. All assembled unigenes were finally annotated by comparing their sequences against the NR (NCBI non-redundant protein sequences, e-value $=$ $10^{-5}$ ), NT (NCBI nucleotide sequences, e-value $\left.=10^{-5}\right)$, SwissProt (e-value $\left.=10^{-5}\right)$, GO (Gene Ontology, e-value $\left.=10^{-6}\right)$, KEGG (Kyoto Encyclopedia of Genes and Genomes, e-value $=$ $10^{-10}$ ), PFAM (protein family, e-value $=0.01$ ), and KOG/COG (euKaryotic Ortholog Groups/ Clusters of Orthologous Groups of Proteins, e-value $=10^{-3}$ ) databases. For NR, SwissProt, and KOG/COG annotations, Diamond v0.8.22 software was used. For NT annotation, Blastn (NCBI blast 2.2.28+) was used. PFAM protein family alignments were performed using the HMMER 3.0 package. GO annotations were obtained based on the annotation results from NR and PFAM using Blast2GO v2.5 software (Götz et al., 2008). The KEGG Automatic Annotation Server of KAAS was used for KEGG pathway analysis. The clean reads were aligned to the Trinity 
transcripts using Bowtie 2 with default parameters (Langmead \& Salzberg, 2012). Following alignment, raw read counts for each transcript were derived using RSEM v1.2.15 (Li \& Dewey, 2011 ) with default parameters and then normalized to FPKM (Fragments Per Kilobase of transcript per Million mapped reads) (Trapnell et al., 2010). The read counts were used as input for the DESeq R package (1.10.1) (Anders \& Huber, 2010) to identify differentially expressed genes (DEGs) using the thresholds adjusted $P$ value $($ padj $)<0.05$ and $\mid \log _{2}$ FoldChange $\mid \geq 1$. DEGs were mapped to terms in the GO and KEGG databases for functional and pathway analysis. The GOseq R package (Young et al., 2010) was used for GO enrichment analysis, and GO terms with corrected $P$ value $<0.05$ were defined as significantly enriched in the DEG set. Significantly enriched KEGG pathways were identified in the DEGs using KOBAS v2.0.12 with a corrected $P$ value $<0.05$ (Mao et al., 2005). The GO and KEGG enrichment maps were generated using the Beijing Novogene Technology Co., Ltd. server. The two figures were merged using Adobe Photoshop CS5 (Adobe Inc.; San Jose, CA, USA).

\section{Verification of RNA-seq gene expression by quantitative real-time PCR}

Samples of total RNA ( $3 \mu \mathrm{g})$ were taken from the same total RNA used for RNA sequencing and used for the synthesis of first-strand cDNA with the M-MLV reverse transcription kit (Takara, Japan) according to the manufacturer's instructions. The cDNA obtained by reverse transcription was diluted 10-fold for use. Primer 5 software was used to design gene-specific real-time quantitative PCR (qRT-PCR) primers. qRT-PCR was performed using the Roche Light Cycler 480 system (Roche, Rotkreuz, Switzerland) and the SYBR Premix ExTaq kit (Takara, Japan) with a $10-\mu \mathrm{L}$ PCR reaction system. The thermal cycle procedure was as follows: pre-incubation at $95^{\circ} \mathrm{C}$ for $5 \mathrm{~min}$, followed by 40 cycles of $95^{\circ} \mathrm{C}$ for $15 \mathrm{~s}, 60^{\circ} \mathrm{C}$ for $15 \mathrm{~s}$, and $72{ }^{\circ} \mathrm{C}$ for $20 \mathrm{~s}$. The $2^{-\Delta \Delta \mathrm{Ct}}$ method was used to calculate relative gene expression using the GAPDH gene as a reference (Livak \& Schmittgen, 2001). Pearson correlations between RNA-seq and qRT-PCR expression fold changes were calculated using SPSS 20.0. Treatment differences were assessed by analysis of variance using SPSS 20.0 (IBM Inc., Chicago, USA).

\section{Data analysis}

All physiological data are presented as the mean \pm standard deviation of three replicates. SPSS 20.0 was used to perform one-way analysis of variance (ANOVA) and correlation analysis, and Tukey's test was used to identify differences among individual treatments $(P<0.05)$. Correlations between biomass and physiological indexes after different durations of salt stress were obtained using the bivariate module in the correlate analysis tool of SPSS 20.0. Graphs were constructed using SigmaPlot 12.5 software.

\section{RESULTS}

\section{Effects of different salt stress levels on the physiological characteristics of Chlorella sp. TLD6B}

Biomass increased under low salt stress and decreased under high salt stress, and Chlorella sp. TLD6B entered the logarithmic growth phase on the sixth day of culture. The $\mathrm{OD}_{680}$ 
231

measurement increased rapidly before day 18 , but slowed by day 24 , entering a stable phase. The $\mathrm{OD}_{680}$ measurement gradually decreased as the concentration of $\mathrm{NaCl}$ added to the medium increased from $0.1 \mathrm{M}$ to $0.8 \mathrm{M}$ (Figure $1 \mathrm{~A}$ and Table S2). The algae were able to tolerate high levels of salt ( $0.6 \mathrm{M}$ and $0.8 \mathrm{M} \mathrm{NaCl}$ addition), but their biomass was very low (Figure $1 \mathrm{~B}$ and Table S2). Biomass was higher in the $0.1 \mathrm{M}$ and $0.2 \mathrm{M} \mathrm{NaCl}$ addition treatments than in the control, but the $\mathrm{OD}_{680}$ measurement decreased with increasing $\mathrm{NaCl}$ concentrations in the remaining treatments and also decreased with time. In general, compared with the control, the $0.1 \mathrm{M}$ and $0.2 \mathrm{M} \mathrm{NaCl}$ addition treatments significantly inhibited the $\mathrm{OD}_{680}$ but increased biomass. Biomass increased markedly after 12 days of $\mathrm{NaCl}$ stress in the $0.2 \mathrm{M} \mathrm{NaCl}$ addition treatment, whereas addition of $0.4-0.8 \mathrm{M} \mathrm{NaCl}$ significantly inhibited both OD680 and biomass.

Carbohydrate content decreased as $\mathrm{NaCl}$ concentration increased: with the exception of the $0.2 \mathrm{M} \mathrm{NaCl}$ addition treatment, the higher the $\mathrm{NaCl}$ concentration in the culture solution, the lower the algal carbohydrate content (Figure 1C and Table S2). However, lipid content and biomass were improved by the addition of up to $0.2 \mathrm{M} \mathrm{NaCl}$. Lipid content as a function of $\mathrm{NaCl}$ concentration was directly proportional to algal biomass, and Chlorella sp. TLD6B exhibited the highest lipid contents of $29.95 \%$ and $31.27 \%$ after the addition of $0.1 \mathrm{M}$ and $0.2 \mathrm{M} \mathrm{NaCl}$, respectively (Figure $1 \mathrm{~B}$ and $1 \mathrm{D}$ and Table S2). When $\mathrm{NaCl}$ concentrations greater than $0.2 \mathrm{M}$ were added, lipid content decreased significantly as salinity increased (Figure 1D and Table S2).

Carbohydrate content gradually increased early in cultivation (days 1-12) but decreased rapidly as total lipid content increased after day 12 . At day 24, carbohydrate contents were $0.74-$, $0.88-, 0.62-, 0.53$-, and 0.38-fold that of the control in the $0.1,0.2,0.4,0.6$, and $0.8 \mathrm{M} \mathrm{NaCl}$ addition treatments, respectively (Figure 1C and Table S2). By contrast, the lipid contents of the same treatments were 1.37-, 1.43-, 1.20-, 1.15-, and 1.13-fold that of the control (Figure 1D and Table S2). At the same time, the stability of the specific growth rate of cells under low salt stress (0.1 and $0.2 \mathrm{M} \mathrm{NaCl}$ addition) was higher than that under medium and high salt stress (0.4-0.8 $\mathrm{M} \mathrm{NaCl}$ addition), resulting in low lipid productivity under high salt stress (Figure $1 \mathrm{E}$ and $1 \mathrm{~F}$ and Table S2). This is consistent with the change in lipid content.

The biomass of Chlorella sp. TLD6B was significantly correlated with its physiological parameters on day 18 of salt stress. Cell density $\left(\mathrm{OD}_{680}\right)$ and carbohydrate content were significantly correlated with biomass on day 18 , but the correlation between biomass and total lipid content was highest $(r=0.79$ ) (Table 1). Therefore, Chlorella cells under low salt stress $(0.1 \mathrm{M} \mathrm{NaCl}$ addition$)$ and high salt stress $(0.8 \mathrm{M} \mathrm{NaCl}$ addition) on day 18 were selected for transcriptome sequencing to further study the mechanisms of lipid accumulation under salt stress.

\section{RNA-sequencing, de novo transcriptome assembly, and functional annotation}

RNA-seq was performed on the mRNA extracted from the two $\mathrm{NaCl}$ treatments and the control, generating 963,078,184 raw reads. A total of 947,225,244 clean reads were obtained after filtering on base quality score and read length. The GC percentage of the clean reads was nearly $66.0 \%$, and the Q20 was greater than 96\% (Table S3). Trinity was used to create a de novo transcriptome assembly from the high-quality clean reads, producing 219,577 transcripts with an 
average length of 1,394 bp. 155,503 non-redundant unigenes were assembled; their length ranged from 200 to 23,825 bp with an average length of 1,842 bp (Tables S4 and S5).

All assembled unigenes were functionally annotated by searching against multiple public databases. Of the 155,503 unigenes, 111,238, 60,439, 84,173, 105,026, 109,098, and 51,484 were successfully annotated in the NR, NT, SwissProt, PFAM, GO, and KOG databases, respectively. Unigenes were also functionally annotated by searching against the KEGG database. A total of 47,032 (30.24\%) unigenes had an annotated function in the KEGG database, and 131,623 (84.64\%) unigenes were successfully annotated in at least one database (Table 2).

\section{DEGs in response to salt stress}

There were a total of 16,402 DEGs across the low salinity $(0.1 \mathrm{M} \mathrm{NaCl}$ addition, Nacl1) and high salinity $(0.8 \mathrm{M} \mathrm{NaCl}$ addition, Nac12) treatments, based on thresholds of $P<0.05$ and $|\log 2 \mathrm{FC}| \geq$ 1 (Figure 2A and Table S6). Of these, 1,326 were up-regulated and 2,304 were down-regulated under low salinity, and 9,776 were up-regulated and 5,798 were down-regulated under high salinity. Eight hundred twenty-eight unigenes were differentially expressed only under low salinity, 12,772 were differentially expressed only under high salinity, and 2,802 were differentially expressed under both conditions (Figure 2B and Table S6). Of the 16,402 total DEGs, $82.9 \%$ were differentially expressed under only the low or the high salinity treatment.

\section{qRT-PCR verification of RNA-seq data}

Nine DEGs in Nacl1 vs. CK18 (the $0.1 \mathrm{M} \mathrm{NaCl}$ treatment compared to the control on day 18) and $\mathrm{Nacl} 2$ vs. $\mathrm{CK} 18$ (the $0.8 \mathrm{M} \mathrm{NaCl}$ treatment compared to the control on day 18) were selected for qRT-PCR verification. These unigenes were mainly involved in fatty acid synthesis, TAG synthesis, the glycolysis/gluconeogenesis pathway, the pentose phosphate pathway, and energy metabolism of the mitochondrial respiratory chain. Seven were up-regulated and two were downregulated. The qRT-PCR primers listed in Table S7 were used to verify the RNA-seq data (Table S7), and the results are shown in Fig. 3A and Table S7. The expression patterns of nine unigenes were similar in the qRT-PCR and RNA-seq data, although there was some deviation in fold change values between the two methods. The qRT-PCR results were highly correlated with the RNA-seq results $\left(r=0.890, r^{2}=0.791\right)$, indicating that the RNA-seq data were generally accurate and reliable (Figure 3B).

\section{GO and KEGG pathway enrichment analysis of the DEGs}

To further explore their functions, the DEGs from the two salt treatments were annotated with GO terms and KEGG pathways for functional and pathway enrichment analyses (Table S6). Among the top 30 most highly enriched GO terms in the 828 specific DEGs from the low salinity treatment, one molecular function (MF) GO term was significantly enriched (corrected $P$ value $<0.05$ ): transferase activity, transferring one-carbon groups (GO:0016741). Among the top 30 most highly enriched GO terms in the 2,802 common DEGs, 10 biological process (BP) terms and $20 \mathrm{MF}$ terms were significantly enriched (corrected $P$ value $<0.05$ ). In the BP category, the three most significantly enriched GO terms were G protein-coupled receptor signaling pathway 
309 (GO:0007186), defense response to other organism (GO:0098542), and response to external

310 biotic stimulus (GO:0043207). The three most significantly enriched MF GO terms were

311 peptidase activity (GO:0008233), peptidase activity, acting on L-amino acid peptides

312 (GO:0070011), and endopeptidase activity (GO:0004175). The most strongly and significantly

313 enriched BP GO term in the 12,772 specific DEGs from the high salinity treatment was

314 transcription initiation from RNA polymerase II promoter (GO:0006367). The three most

315 significantly enriched cellular component (CC) GO terms were RNA polymerase II transcription

316 factor complex (GO:0090575), nuclear transcription factor complex (GO:0044798), and

317 transcription factor TFIIA complex (GO:0005672). The most significantly enriched MF GO term

318 was secondary active transmembrane transporter activity (GO:0015291) (Figure 4 and Table S8).

319 There were no significantly enriched KEGG pathways in the low salinity-specific DEGs. Among

320 the top 20 KEGG pathways enriched in the common DEGs, seven were significantly enriched

321 (corrected $P$ value $<0.05$ ): plant hormone signal transduction (ko04075), arginine and proline metabolism (ko00330), glutathione metabolism (ko00480), betalain biosynthesis (ko00965), sulfur metabolism (ko00920), cysteine and methionine metabolism (ko00270), and nitrogen metabolism (ko00910). The 12,772 high salinity-specific DEGs were significantly enriched in mismatch repair (ko03430), DNA replication (ko03030), nucleotide excision repair (ko03420), proteasome (ko03050), photosynthesis (ko00195), porphyrin and chlorophyll metabolism (ko00860), and galactose metabolism (ko00052) (Figure 5 and Table S9). Consistent with the KEGG analysis, GO enrichment analysis showed that common DEGs under low and high salinity were involved primarily in stress response, defense response, and membrane structure.

\section{Transcriptional expression of unigenes involved in fatty acid and TAG biosynthesis pathways}

\section{Fatty acid biosynthesis}

Acetyl-CoA, the precursor for fatty acid synthesis in the cytosol, derives from pyruvate (PYR) produced in glycolysis by pyruvate kinase (PK). Genes encoding two rate-limiting enzymes in glycolysis, hexokinase (HXK) and PK, were up-regulated under both the high and low salinity treatments, and 6-phosphofructokinase (PFK) was up-regulated under the high salinity treatment (Figure 6 and Table S10). These changes would have promoted the accumulation of acetyl-CoA and energy.

The expression of key genes of the de novo fatty acid synthesis pathway also changed in response to salinity treatment. For example, the acetyl CoA carboxylase (ACCase) genes $B C$ and $B C C P$ were up-regulated on day 18 under both salinity treatments. The $\alpha-C T$ gene (Cluster31803.29741) was up-regulated 2.7-fold under high salinity (Figure 7A and Table S11), and the malonyl-CoA:ACP transacylase (MCTA) gene (Cluster-31803.71595) was also up-regulated only under high salinity (Figure 7B and Table S11). Two 3-oxoacyl-[acyl carrier protein] synthase II (KAS II) genes (Cluster-31803.17167 and Cluster-31803.96146) were up-regulated under both salinity treatments, but these differences were not significant (Figure $7 \mathrm{C}$ and Table S11). One $\omega$ 3 fatty acid desaturase $(F A D)$ gene (Cluster-31803.83020) was down-regulated 4.5-fold under 
348 high salinity, and the other FAD gene (Cluster-31803.96909) was up-regulated 1.5-fold under

349 low salinity (Figure 7D and Table S11). A stearoyl ACP desaturase (SAD) gene (Cluster-

35031803.15683 ) was down-regulated under low salinity, whereas three $S A D$ genes were up-

351 regulated under high salinity (Cluster-31803.73844, Cluster-31803.73842, and Cluster-

352 31803.73850) (Figure 7E and Table S11).

\section{TAG biosynthesis}

354 Four NAD-3-phosphate glycerol dehydrogenase $(G P D)$ genes and one glycerol kinase $(G K)$ gene

355

356

357

358

359

360

361

362

363

364

365

366

367

368

369

370

371

372

373

374

375

376

377

378

379

380

381

382

383

384

were significantly up-regulated under high salinity. A gene encoding the glycolysis enzyme triose-phosphate isomerase (TPI) was up-regulated under both salinity treatments, which may have resulted in the accumulation of DHAP (Figures 6,8A, 8B, and $8 \mathrm{C}$ and Table S12) and promoted the synthesis of TAG. One DGAT1 gene and seven DGAT2 genes were identified in our transcriptome data. All but two were up-regulated (Cluster-31803.65991 and Cluster31803.86613) (Figures 6, 8D and Table S12).

\section{Expression of key unigenes related to lipid synthesis pathways}

To further explore mechanisms of lipid accumulation in Chlorella sp. TLD6B under salt stress, we compared the expression of genes associated with the metabolic pathways of starch-lipid biosynthesis. Under high salinity, a single gene encoding the starch-degrading enzyme $\alpha$-amylase (AMY3, Cluster-31803.62982) was up-regulated 4.6-fold (Figure 6 and Table S10).

Furthermore, genes encoding ACL, PDC/PDH, ME, G6PD, and PEPC were primarily upregulated. All of these genes showed greater expression under high salinity than under low salinity (Figure 6 and Table S10). The upregulation of these genes whose products function in central carbon metabolism may directly or indirectly promote the accumulation of fatty acids.

\section{DISCUSSION}

High salinity is generally not conducive to the growth and development of organisms because of $\mathrm{Na}^{+}$toxicity, excessive ROS accumulation, and difficulties related to osmotic balance and water absorption. The salt tolerance mechanisms of different microalgae may differ. To date, many studies have shown that salt treatment strongly promotes lipid synthesis in microalgae but also inhibits cell growth (Sharma et al., 2012; Fan et al., 2014; Kato et al., 2017; Li et al., 2018). According to current research, marine microalgae have higher salt tolerance than other algae such as Dunaliella salina (Takagi et al., 2006; Benmoussa-Dahmen et al., 2016). By analyzing salt resistance and lipid content, we found that Chlorella sp. TLD6B could tolerate high levels of salt stress (0.8 M NaCl addition) that are lethal to many other freshwater algae (Li et al., 2018). Chlorella sp. TLD6B showed greater lipid accumulation following addition of 0.1 and $0.2 \mathrm{M}$ $\mathrm{NaCl}$, but this effect decreased at higher salt levels. This result indicates that Chlorella sp. TLD6B has a mechanism for increasing lipid accumulation under salt stress. By extension, the addition of 0.1 or $0.2 \mathrm{M} \mathrm{NaCl}$ can significantly promote Chlorella sp. TLD6B lipid accumulation and potentially reduce the production costs of biodiesel. 
The salinity-induced accumulation of lipids in microalgae begins with a signal transduction cascade initiated by the perception of a salt stress signal. Downstream results include an increase in lipid and unsaturated fatty acid concentrations, the rapid accumulation of solutes that regulate cellular osmotic balance (soluble sugars, amino acids, and betaine), and the induction of the ROS scavenging system (Ramos et al., 2011). Here, we found that many key genes involved in glycerol, fatty acid, and lipid metabolism were significantly up-regulated in response to different salinity treatments, suggesting that the salt tolerance of Chlorella sp. TLD6B is related to its lipid metabolism. Low levels of $\mathrm{NaCl}$ addition up-regulated the expression of genes related to fatty acid synthesis $(B C, B C C P, K A S I I)$ and promoted increased biomass and lipid production, indicating that low salinity is essential for the growth of Chlorella sp. TLD6B and ultimately promotes its lipid accumulation. Nonetheless, the regulation of lipid accumulation through the manipulation of salinity levels requires further study. Rao et al. (2007) reported that increased salt concentration in the growth medium altered the membrane lipid composition of Botryococcus braunii LB 572 cells: the proportion of stearic and linoleic acid decreased, and that of oleic acid rose. These results suggested that unsaturated fatty acids play a role in salt tolerance.

Previous studies have also shown that SAD is a key enzyme for the synthesis and metabolism of unsaturated fatty acids, directly determining the total amount of unsaturated fatty acids in vegetable oils as well as the ratio of saturated to unsaturated fatty acids (Kachroo et al., 2007). FAD is considered to be a candidate gene for the promotion of high oleic acid levels (Jung et al., 2000a). Schwartzbeck et al. (2001) used transgenic technology to inhibit FAD2 enzyme activity and increase the oleic acid content of corn oil from $25 \%$ to $64 \%$. Jung et al. (2000b) increased the ratio of oleic acid to linoleic acid in peanuts by inhibiting FAD2 enzyme activity. Here, we also found that high salinity up-regulated $S A D$ expression in Chlorella sp. TLD6B, whereas low salinity down-regulated $S A D$ expression. One $F A D$ gene (Cluster31803.83020) was down-regulated 4.5-fold under high salinity, whereas another (Cluster31803.96909) was up-regulated 1.5-fold under low salinity (Figure 7D). This indicates that $S A D$ and $F A D$ may be involved in the regulation of fatty acid components in Chlorella sp. TLD6B. Low salinity may promote fatty acid synthesis, whereas high salinity may enhance unsaturated fatty acid synthesis, thereby reducing damage to cell membranes during salt stress (Kan et al., 2012). The lipid content of Chlorella sp. TLD6B increases under high salinity, and it is unknown whether the unsaturated fatty acid content also increases. Although we did not measure fatty acid composition, the transcriptome data indicated that $S A D$ genes were up-regulated and an $F A D$ gene was down-regulated under high salinity. This result suggests that the unsaturated fatty acid content may increase under high salt stress to reduce cell membrane damage.

Our results revealed that the pathway of lipid synthesis in Chlorella sp. TLD6B is finely regulated in response to different salinity conditions. First, upregulation of the key glycolysis genes $H X K$ and $P K$, which implies enhancement of pentose phosphate metabolism, was likely to promote rapid acetyl-CoA accumulation and a stable intracellular energy supply. Therefore, high glycolysis rates may be one reason for the high lipid accumulation observed in Chlorella sp. 
425 TLD6B in response to $\mathrm{NaCl}$ addition. Second, ACCase catalyzes the formation of malonyl-CoA 426 from acetyl-CoA and is the primary rate-limiting enzyme for de novo fatty acid synthesis. 427 Upregulation of the corresponding gene may increase the rate of fatty acid synthesis (Roesler et 428 al., 1997; Zalutskaya et al., 2015). We found that key genes of fatty acid synthesis, ACCase (BC 429 and $B C C P$ ) and $K A S I I$, were up-regulated after the addition of $\mathrm{NaCl}$ (Figure 7A, 7C), which 430 may also have enhanced fatty acid synthesis. Moreover, $A C L, P D C / P D H$, and $M E$ in the PDH 431 bypass were up-regulated, which may have accelerated the accumulation of precursor substrates 432 and energy for fatty acid biosynthesis (Tan \& Lee, 2016). The presence and high expression of 433 these genes suggest that Chlorella sp. TLD6B uses a specific approach to ensure the rapid 434 biosynthesis of fatty acids and TAG. Finally, the fatty acyl-CoA produced by de novo synthesis 435 is esterified to produce TAG. The synthesis of TAG requires not only fatty acids but also 436 glycerol 3-phosphate (G3P) (Ohlrogge \& Browse, 1995). G3P is synthesized from glycerol by 437 glycerol kinase (GK) or from DHAP by NAD-3-phosphate glycerol dehydrogenase (GPD) (Yao 438 et al., 2014; Huang et al., 2016) (Figures 6, 8B and 8C). In addition, research has shown that the overexpression of GPDH in microalgae promotes the conversion of DHAP to G3P (Yao et al., 2014) and that overexpression of fatty acid synthesis genes such as ACCase increases lipid accumulation (Courchesne et al., 2009). Both of these pathways compete for precursor substrates, and the flux of carbon out of glycolysis towards glycerol formation can cause cell growth to be inhibited. In our transcriptome data, four GPD genes and one $G K$ gene were significantly up-regulated under high salt stress, and two TPI genes were up-regulated under both low and high salinity; these changes are beneficial to TAG synthesis but may inhibit cell growth. Therefore, salt stress only moderately increases the lipid concentration. This may explain why, in our study, carbohydrate levels gradually declined under salt stress while lipid accumulation increased. Moreover, the total lipid concentration under high salinity decreased to that of the control and was lower than the total lipid concentration under low salinity (Figure 1D).

Therefore, we suggest that salt stress alters the carbon flow between starch biosynthesis and lipid biosynthesis in Chlorella sp. TLD6B.

In addition, recent transcriptome studies have shown that microalgal lipid synthesis may be influenced by the conversion between starch and lipid synthesis (Shang et al., 2016; Ho et al., 2017). Here, only one AMY3 gene (Cluster-31803.62982) was up-regulated 4.6-fold under high salinity. Lipid synthesis in Chlorella sp. TLD6B may therefore not be closely related to the conversion between starch and lipid biosynthesis. Its lipid accumulation may be controlled primarily by glycolysis and the synthesis of fatty acids and TAG.

\section{CONCLUSION}

We performed physiological measurements and genome-wide transcriptome profiling of Chlorella sp. TLD6B under different levels of salt stress. Chlorella sp. TLD6B was able to tolerate high salt stress $(0.8 \mathrm{M} \mathrm{NaCl}$ addition). Lipid concentration first increased and then decreased with increasing levels of salt stress, reaching a maximum under the addition of $0.2 \mathrm{M}$ $\mathrm{NaCl}$. Comparative transcriptomic analysis revealed that salt stress enhanced the expression of genes related to sugar metabolism and fatty acid biosynthesis (the ACCases BC and BCCP, KAS 
$465 I I$, and GPDHs involved in TAG synthesis), thereby promoting lipid accumulation under the

466 addition of $0.2 \mathrm{M} \mathrm{NaCl}$. However, high salt stress also inhibited cell growth. Expression of three

$467 S A D s$, whose encoded products function in unsaturated fatty acid biosynthesis, was up-regulated 468 under high salinity $(0.8 \mathrm{M} \mathrm{NaCl}$ addition $)$. This research clarifies the relationship between salt 469 tolerance and lipid accumulation, promoting the development and utilization of Chlorella $\mathrm{sp}$.

470 TLD6B for biofuel production.

471 ADDITIONAL INFORMATION AND DECLARATIONS

472 Funding

473 This study was supported by the National Natural Science Foundation of China (31460276) and 474 the Shihezi University independently funded Project (ZZZC201840B). The funders had no role 475 in study design, data collection and analysis, decision to publish, or preparation of the 476 manuscript.

\section{Grant Disclosures}

478 The following grant information was disclosed by the authors:

479 National Natural Science Foundation of China: 31460276.

480 Shihezi University independently funded Project: ZZZC201840B.

481 Competing Interests

482 The authors declare there are no competing interests.

\section{Author Contributions}

- Hong Li performed the experiments, analyzed the data, prepared the figures and/or tables, and wrote and reviewed drafts of the paper.

- Jun Tan performed experiments and prepared figures and/or tables.

- Yun Mu performed experiments, prepared figures and/or tables, and analyzed the data.

- Jianfeng Gao conceived and designed the experiment, reviewed drafts of the paper, and approved the final draft.

\section{Data Availability}

The following information was supplied regarding data availability: Chlorella sp. TLD6B transcriptome sequence data are available at NCBI GEO under accession numbers: GSE162916. Raw data are available in the Supplemental Files.

\section{Acknowledgments}

The authors would like to thank TopEdit (www.topeditsci.com) for linguistic assistance during preparation of this manuscript.

Supplemental Information

498 Supplemental information for this article can be found online at ***. 


\section{REFERENCES}

Anders S, Huber W. 2010. Differential expression analysis for sequence count data. Genome Biology 11(10):R106 DOI 10.1186/gb-2010-11-10-r106.

Ashley J, Rushforth, SR. 1984. Growth of soil algae on top soil and processed oil shale from the Uintah Basin, Utah, U.S.A. Reclamation and Revegatation Research 3:49-63 DOI 10.1007/BF01407604.

BenMoussa-Dahmen I, Chtourou H, Rezgui F, Sayadi S, Dhouib A. 2016. Salinity stress increases lipid, secondary metabolites and enzyme activity in Amphora subtropica and Dunaliella sp. for biodiesel production. Bioresource Technology 218:816-825 DOI 10.1016/j.biortech.2016.07.022.

Courchesne NMD, Parisien A, Wang B, Lan CQ. 2009. Enhancement of lipid production using biochemical, genetic and transcription factor engineering approaches. Journal of Biotechnology 141(12):31-41 DOI 10.1016/j.jbiotec.2009.02.018.

Davidson NM, Oshlack A. 2014. Corset: enabling differential gene expression analysis for de novo assembled transcriptomes. Genome Biology 15(7):410 DOI 10.1186/s13059-014-0410-6.

Dubois M, Gilles HA, Hamilton JK, Rebers PA. 1956. Colorimetric method for determination of sugars and related substances. Analytical Chemistry 28(3):22-25 DOI 10.1021/ac60111a017.

Fan JH, Cui YB, Wan MX, Wang WL, Li YG. 2014. Lipid accumulation and biosynthesis genes response of the oleaginous Chlorella pyrenoidosa under three nutrition stressors. Biotechnology for Biofuels 7:17 DOI 10.1186/1754-6834-7-17.

Gao CF, Wang Y, Shen Y, Yan D, He X, Dai JB. Wu QY. 2014. Oil accumulation mechanisms of the oleaginous microalga chlorella protothecoides revealed through its genome, transcriptomes, and proteomes. BMC Genomics 15:582 DOI 10.1186/1471-2164-15-582.

Garcia-Pichel F, Lopez-Cortes A, Nubel U. 2001. Phylogenetic and morphological diversity of cyanobacteria in soil desert crusts from the Colorado Plateau. Applied and Environmental Microbiology 67:1902-1910 DOI 10.1128/AEM.67.4.1902-1910.2001.

Gong CX, Li FF, Gou YF, Wang D, Gao JF. 2013. Comparison of different culture methods for a desert chlorella. Renewable Energy Resources 31(11):106-115.

Grabherr MG, Haas BJ, Yassour M, Levin JZ, Thompson DA, Amit I, Adiconis X, Fan L, Raychowdhury R, Zeng Q, Chen Z, Mauceli E, Hacohen N, Gnirke A, Rhind N, Palma FD, Birren BW, Nusbaum C, Lindblad-Toh K, Friedman N, Regev A. 2011. Full-length transcriptome assembly from RNA-Seq data without a reference genome. Nature Biotechnology 29:644-652 DOI 10.1038/nbt.1883.

Götz S, García-Gómez JM, Terol J, Williams TD, Nagaraj SH, Nueda MJ, Robles M, Robles M, Dopazo J, Conesa A. 2008. High-throughput functional annotation and data mining with the Blast2GO suite. Nucleic Acids Research 36:3420-3435 DOI 10.1093/nar/gkn176.

Halim R, Danquah MK, Webley PA. 2012. Extraction of oil from microalgae for biodiesel production: A review. Biotechnology Advances 30:709-732 DOI 10.1016/j.biotechadv.2012.01.001.

Ho SH, Nakanish A, Ye X, Chang JS, Hara K, Hasunuma T, Kondo A. 2014. Optimizing biodiesel production in marine Chlamydomonas sp. JSC4 through metabolic profiling and an innovative salinitygradient strategy. Biotechnology for Biofuels 7:97 DOI 10.1186/1754-6834-7-97.

Ho SH, Nakanishi A, Kato Y, Yamasaki H, Chang JS, Misawa N, Hirose Y, Minagawa J, Hasunuma T, Kondo A. 2017. Dynamic metabolic profiling together with transcription analysis reveals salinityinduced starch-to-lipid biosynthesis in alga chlamydomonas sp. JSC4. Scientific Reports 7:45471 DOI 10.1038/srep45471.

Huang WP, Ye JR, Zhang JJ, Lin Y, He MX, Huang JC. 2016. Transcriptome analysis of chlorella 
zofingiensis to identify genes and their expressions involved in astaxanthin and triacylglycerol biosynthesis. Algal Research 17:236-243 DOI 10.1016/j.algal.2016.05.015.

Jung S, Powell G, Moore K, Abbott A. 2000a. The high oleate trait in the cultivated peanut (Arachis hypogaea L.). II. Molecular basis and genetics of the trait. Molecular Genetics and Genomics 263(5):806811 DOI 10.1007/s004380000243.

Jung S, Swift D, Sengoku E, Patel M, Teulé F, Powell G, Moore K, Abbott A. 2000b. The high oleate trait in the cultivated peanut (Arachis hypogaea L.). I. Isolation and characterization of two genes encoding microsomal oleoyl-PC desaturases. Molecular \& General Genetics Mgg 263(5):796-805 DOI $10.1007 / \mathrm{s} 004380000244$.

Kachroo A, Shanklin J, Whittle E, Lapchyk L, Hildebrand D, Kachroo P. 2007. The Arabidopsis stearoylacyl carrier protein-desaturase family and the contribution of leaf isoforms to oleic acid synthesis. Plant Molecular Biology 63(2):257-271 DOI 10.1007/s11103-006-9086-y.

Kan GF, Shi CJ, Wang XF, Xie QJ, Wang M, Wang XL, Miao JL. 2012. Acclimatory responses to highsalt stress in Chlamydomonas (Chlorophyta, Chlorophyceae) from Antarctica. Acta Oceanologica Sinica 31(01):116-124 DOI CNKI:SUN:SEAE.0.2012-01-015.

Kato Y, Ho SH, Vavricka CJ, Chang JS, Hasunuma T, Kondo A. 2017. Evolutionary engineering of saltresistant, chlamydomonassp. strains reveals salinity stress-activated starch-to-lipid biosynthesis switching. Bioresource Technology 245:1484-1490 DOI 10.1016/j.biortech.2017.06.035.

Langmead B, Salzberg SL. 2012. Fast gapped-read alignment with Bowtie 2. Nat Methods 9(4):357-9 DOI 10.1038/nmeth.1923.

Lewis LA, Flechtner VR. 2002. Green algae (Chlorophyta) of Desert microbiotic crusts: Diversity of North American Taxa. Taxon 51(3): 443-451 DOI 10.2307/1554857.

Li B, Dewey CN. 2011. RSEM: accurate transcript quantification from RNA-Seq data with or without a reference genome. BMC Bioinformatics 12:323 DOI 10.1186/1471-2105-12-323.

Li FF, Gong CX, Zuo XX, Chen F, Gao JF. 2012. Crude lipid content and fatty acid composition of six strains of green microalgae in Gurbantunggut desert. China Oils And Fats 37(12):74-77 DOI 10.3969/j.issn.1003-7969.2012.12.019.

Li L, Zhang G, Wang Q. 2016. De novo transcriptomic analysis of Chlorella sorokiniana reveals differential genes expression in photosynthetic carbon fixation and lipid production. BMC Microbiology 16:223 DOI 10.1186/s12866-016-0839-8.

Li PL, Miao XL, Li RX, Zhong JJ. 2011. In situ biodiesel production from fast-growing and high oil content Chlorella pyrenoidosa in rice straw hydrolysate. Journal of Biomedicine \& Biotechnology 7:141207 DOI 10.1155/2011/141207.

Li XY, Yuan YZ, Cheng DJ, Gao J, Kong LZ, Zhao QY, Wei W, Sun YH. 2018. Exploring stress tolerance mechanism of evolved freshwater strain Chlorella $\mathrm{sp}$. S30 under $30 \mathrm{~g} / \mathrm{L}$ salt. Bioresource Technology 250:495-504 DOI 10.1016/j.biortech.2017.11.072.

Liang YN, Sarkany N, Cui Y. 2009. Biomass and lipid productivities of Chlorella vulgaris under autotrophic, heterotrophic and mixotrophic growth conditions. Biotechnology Letters 31(7):1043-1049 DOI 10.1007/s10529-009-9975-7.

Liu J, Huang JC, Sun Z, Zhong YJ, Jiang Y, Chen F. 2011. Differential lipid and fatty acid profiles of photoautotrophic and heterotrophic Chlorella zofingiensis: Assessment of algal oils for biodiesel production. Bioresource Technology 102(1):106-110 DOI 10.1016/j.biortech.2010.06.017.

Livak KJ, Schmittgen TD. 2001. Analysis of relative gene expression data using real-time quantitative PCR and the 2(-Delta Delta C(T)) Method. Methods 25(4):402-408 DOI 10.1006/meth.2001.1262. 
Mao XZ, Cai T, Olyarchuk JG, Wei LP. 2005. Automated genome annotation and pathway identification using the KEGG Orthology (KO) as a controlled vocabulary. Bioinformatics 21(19):3787-3793 DOI $10.2307 / 1592215$.

Markou G, Nerantzis E. 2013. Microalgae for high-value compounds and biofuels production: A review with focus on cultivation under stress conditions. Biotechnology Advances 31(8):1532-1542 DOI 10.1016/j.biotechadv.2013.07.011.

Mishra SK, Suh WI, Farooq W, Moon M, Shrivastav A, Park MS,Yang JW. 2014. Rapid quantification of microalgal lipids in aqueous medium by a simple colorimetric method. Bioresource Technology 155:330333 DOI 10.1016/j.biortech.2013.12.077.

Mu Y, Wang WL, Li H, Yan G, Wang HM, Gao JF. 2016. Effects of PEG6000 on growth and lipid accumulation of desert chlorella. China Oils and Fats 41(10):58-61.

Nichols HW. 1973. Growth media - freshwater. In: Stein, J. R. (Ed.), Handbook of Phycological Methods, Culture methods and growth measurements. Cambridge University Press pp.7-24.

OhIrogge J, Browse J. 1995. Lipid biosynthesis. Plant Cell 7:957-970 DOI 10.1105/tpc.7.7.957.

Ramos AA, Polle J, Tran D, Cushman JC, Varela JC. 2011. The unicellular green alga Dunaliella salina Teod. as a model for abiotic stress tolerance: genetic advances and future perspectives. Algae 26(1):3-20 DOI 10.4490/algae.26.1.003.

Rao AR, Dayananda C, Sarada R, Shamala TR, Ravishankar GA. 2007. Effect of salinity on growth of green alga Botryococcus braunii and its constituents. Bioresource Technology 98:560-564 DOI 10.1016/j.biortech.2006.02.007.

Roesler K, Shintani D, Savage L, Boddupalli S, Ohlrogge J. 1997. Targeting of the Arabidopsis homomeric acetyl-coenzyme A carboxylase to plastids of rapeseeds. Plant physiology 113(1):75-81 DOI 10.1104/pp.113.1.75.

Schenk PM, Thomas-Hall SR, Stephens E, Marx UC, Mussgnug JH, Posten C, Kruse O, Hankamer B. 2008. Second Generation Biofuels: High-Efficiency Microalgae for Biodiesel Production. BioEnergy Research 1:20-43 DOI 10.1007/s12155-008-9008-8.

Schwartzbeck JL, Jung S, Abbott AG, Mosley E, Lewis S, Pries GL, Powellg L. 2001. Endoplasmic oleoyl-PC desaturase references the second double bond. Phytochemistry 57(5):643-652 DOI 10.1016/s0031-9422(01)00081-4.

Shang CH, Bi GC, Yuan ZH, Wang ZM, Alam MA, Xie J. 2016. Discovery of genes for production of biofuels through transcriptome sequencing of Dunaliella parva. Algal Research 13:318-326 DOI 10.1016/j.algal.2015.12.012.

Sharma KK, Schuhmann H, Schenk PM. 2012. High lipid induction in Microalgae for Biodiesel Production. Energies 5(5):1532-1553 DOI 10.3390/en5051532.

Sharma T, Chauhan RS. 2016. Comparative transcriptomics reveals molecular components associated with differential lipid accumulation between microalgal sp. Scenedesmus dimorphus and Scenedesmus quadricauda. Algal Research 19:109-122 DOI 10.1016/j.algal.2016.07.020.

Srivastava G, Nishchal, Goud VV. 2017. Salinity induced lipid production in microalgae and cluster analysis (iccb 16-br_047). Bioresource Technology 242:244-252 DOI 10.1016/j.biortech.2017.03.175.

Takagi M, Karseno, Yoshida T. 2006. Effect of salt concentration on intracellular accumulation of lipids and triacylglyceride in marine microalgae Dunaliella cells. Journal of Bioence and Bioengineering 101(3):223-226 DOI 10.1263 / jbb.101.223.

Tan KW, Lee YK. 2016. The dilemma for lipid productivity in green microalgae: importance of substrate provision in improving oil yield without sacrificing growth. Biotechnology for Biofuels 9:255 DOI 10.1186/s13068-016-0671-2. 
633

634

635

636

637

638

639

640

641

642

643

644

645

646

647

648

649

650

651

652

653

654

655

656

657

658

659

660

Trapnell C, Williams BA, Pertea G, Mortazaviet A, Kwan G, van Baren MJ, Salzberg SL, Wold BJ, Pachter L. 2010. Transcript assembly and quantification by RNA-Seq reveals unannotated transcripts and isoform switching during cell differentiation. Nature Biotechnology 28(5):511-515 DOI $10.1038 /$ nbt.1621.

Yao Y, Lu Y, Peng KT, Huang T, Niu YF, Xie WH, Yang WD, Liu JS, Li HY. 2014. Glycerol and neutral lipid production in the oleaginous marine diatom Phaeodactylum tricornutum promoted by overexpression of glycerol-3-phosphate dehydrogenase. Biotechnology for Biofuels 7(1):1-9 DOI 10.1186/1754-6834-7-110.

Young MD, Wakefield MJ, Smyth GK, Oshlack A. 2010. Gene ontology analysis for RNA-seq: accounting for selection bias. Genome Biol 11(2):R14 DOI 10.1186/gb-2010-11-2-r14.

Wan MX, Zhang Z, Wang J, Huang JK, Fan JH, Yu AQ, Wang WL, Li YG. 2015. Sequential heterotrophy-dilution-photoinduction cultivation of haematococcus pluvialis for efficient production of astaxanthin. Bioresource Technology 198:557-563 DOI 10.1016/j.biortech.2015.09.031.

Wang D, Gong, CX, Gou YF, Zhu L, Zhu JB, Gao JF. 2014. Phylogenetic analyses on the biological crusts of several algae in the Taklimakan Desert. Acta Prataculturae Sinica 23(03):97-103.

Wang T, Ge HY, Liu TT, Tian XW, Wang ZJ, Guo MJ, Chu J, Zhuang YP. 2016. Salt stress induced lipid accumulation in heterotrophic culture cells of chlorella protothecoides: Mechanisms based on the multilevel analysis of oxidative response, key enzyme activity and biochemical alteration. Journal of Biotechnology 228:18-27 DOI 10.1016/j.jbiotec.2016.04.025.

Zalutskaya Z, Kharatyan N, Forchhammer K, Ermilova E. 2015. Reduction of PII signaling protein enhances lipid body productionin Chlamydomonas reinhardtii. Plant Science 240:1-9 DOI 10.1016/j.plantsci.2015.08.019.

Zhao JC, Zhang BC, Zhang YM. 2006. Study on Chlorophytes of Microbiotic Crusts in the Gurbantonggut Desert, Xinjiang. Arid Zone Research 23(2):189-194 DOI 10.13866/j.azr.2006.02.001.

Zhang JT, Wang MZ, He Q, Pan HL, Meng L, Wang YH. 2020. Variation characteristics of nocturnal lowlevel jet in summer over the hinterland of Taklimakan Desert. Journal of Desert Research 40(5):89-100.

Zhu JB, Wang D, Gou YF, Wang WL, Gao JF. 2014. Screening of microalgae rich in lipid in Xinjiang desert. China Oils and Fats 39(12):68-71 DOI 10.3969/j.issn.1003-7969.2014.12.020.

PeerJ reviewing PDF | (2020:12:56003:3:0:NEW 29 Apr 2021) 


\section{Figure 1}

Figure 1 Effects of different levels of salt stress on the growth, biomass, carbohydrate content, lipid content, specific growth rate, and lipid productivity of Chlorella sp. TLD6B.

(A) Growth curves of Chlorella sp. TLD6B under different levels of salt stress. (B) Biomass of Chlorella sp. TLD6B under different levels of salt stress. (C) Carbohydrate content of Chlorella sp. TLD6B under different levels of salt stress. (D) Lipid content of Chlorella sp. TLD6B under different levels of salt stress. (E) Specific growth rate of Chlorella sp. TLD6B under different levels of salt stress. (F) Lipid productivity of Chlorella sp. TLD6B under different levels of salt stress. DW, dry weight. Bars represent SD.
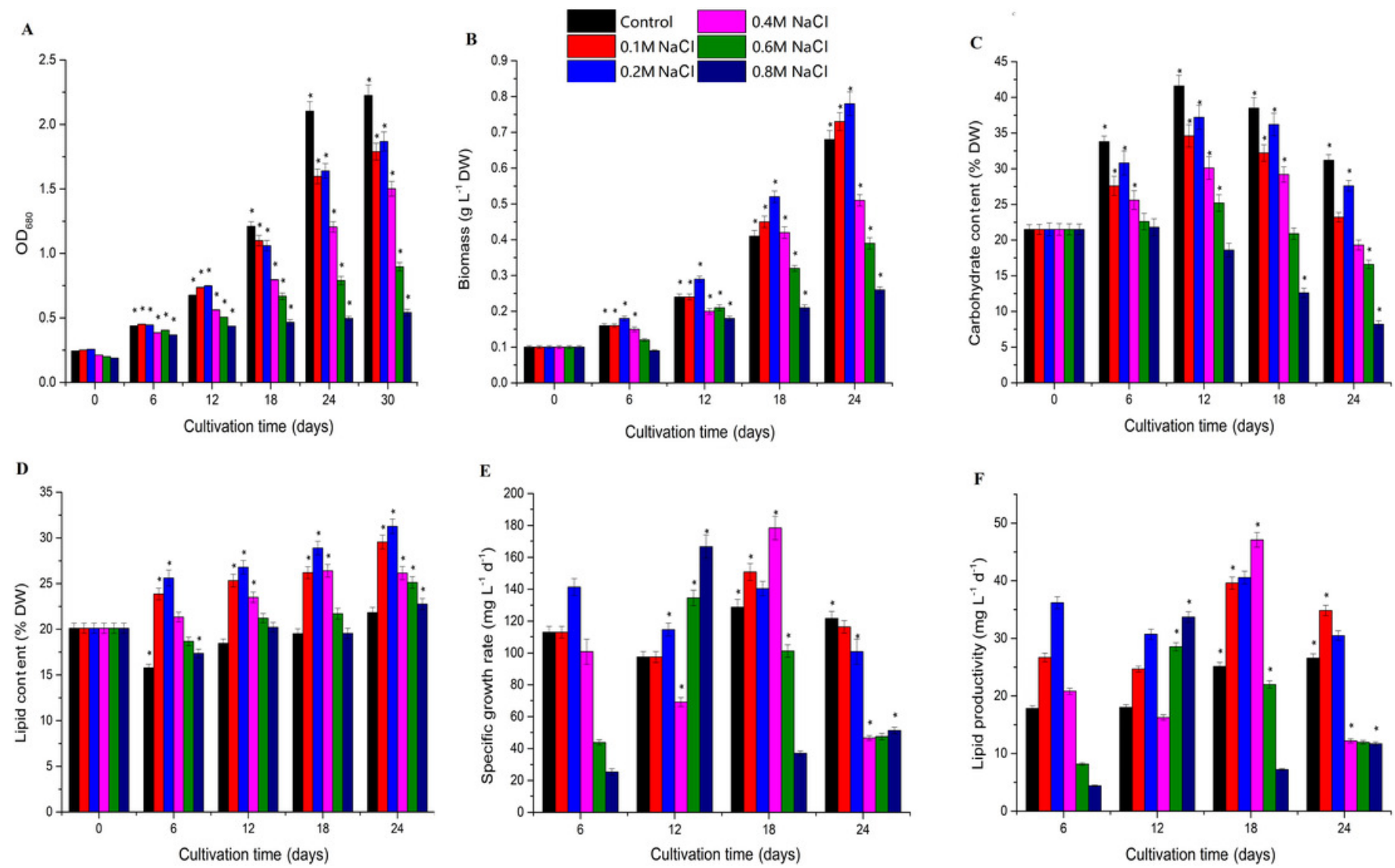
Figure 2

Figure 2 DEGs in the $\mathrm{Nacl} 1(0.1 \mathrm{M} \mathrm{NaCl}$ addition) and $\mathrm{Nacl} 2(0.8 \mathrm{M} \mathrm{NaCl}$ addition) treatments.

(A) A Venn diagram of DEGs in the Nacl1 and Nacl2 treatments. (B) Numbers of up- and downregulated genes in the Nacl1 and Nacl2 treatments.
A
B 12000
Up-regulated

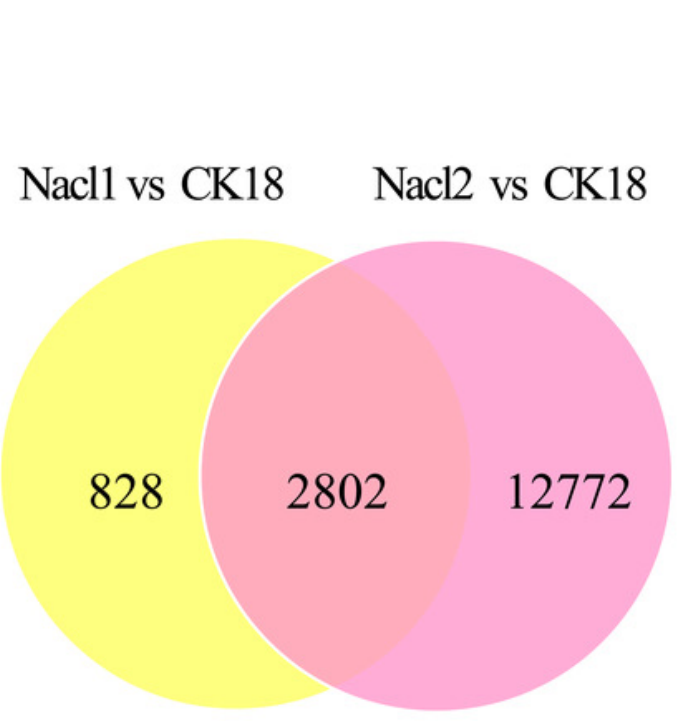

10000
Down-regulated
9776
8000
6000
5798
4000
2000
4000
0
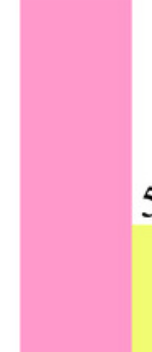
2304
1326
1326
Nacl1 vs CK18
Nacl2 vs CK18 


\section{Figure 3}

Figure 3 qRT-PCR verification of RNA sequencing results.

(A) Comparison of log2 (fold change) values detected by RNA-seq and qRT-PCR. (B) Correlation between RNA-seq and qRT-PCR values. Log2FC, $\log _{2}$ (fold-change) in gene expression between two groups. BCCP, biotin carboxyl carrier protein of acetyl-CoA carboxylase; KAS II, 3-oxoacyl-[acyl-carrier-protein] synthase II; TPI, triose-phosphate isomerase; PK, pyruvate kinase; PEPC, phosphoenolpyruvate carboxylase; PDC/PDH, pyruvate dehydrogenase; G6PD, glucose-6-phosphate dehydrogenase; 6PGDH, 6-phosphogluconate dehydrogenase; GAPD, glyceraldehyde 3-phosphate dehydrogenase.
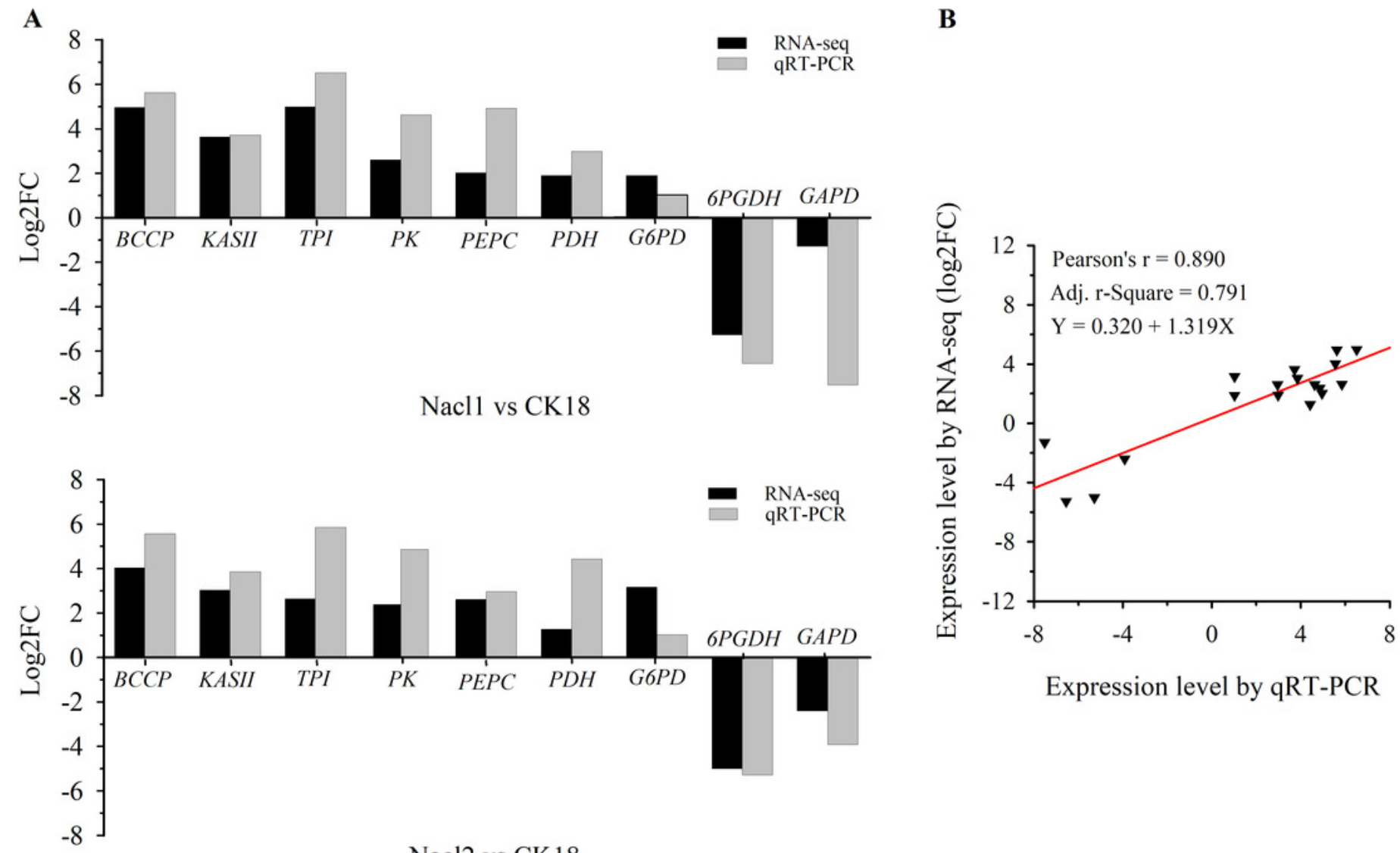

Nacl2 vs CK18 


\section{Figure 4}

Figure $4 \mathrm{GO}$ enrichment of DEGs under salt stress.

(A) The top 30 most highly enriched GO terms in 828 unigenes specifically differentially expressed under low salinity (0.1 M NaCl addition); (B) The top 30 most highly enriched GO terms in 12,772 unigenes specifically differentially expressed under high salinity $(0.8 \mathrm{M} \mathrm{NaCl}$ addition); and (C) the top 30 most highly enriched GO terms in 2,802 unigenes differentially expressed under both high and low salinity. The $x$-axis indicates the number of unigenes in each secondary GO classification. The y-axis indicates the secondary GO classification, and the three colors represent the three major types of $\mathrm{GO}$ terms (i.e., BP, CC, and MF). *corrected $p$-value $<0.05$. 
A

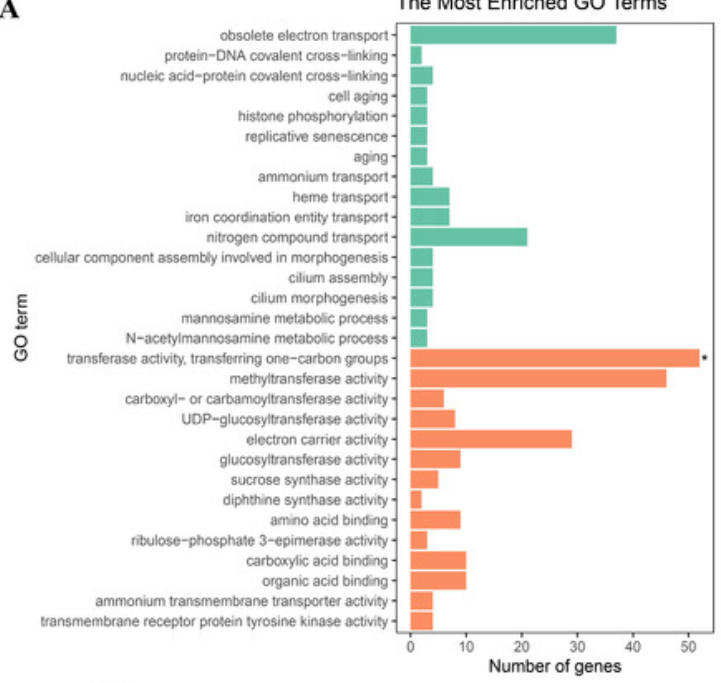

C

छ

The Most Enriched GO Terms

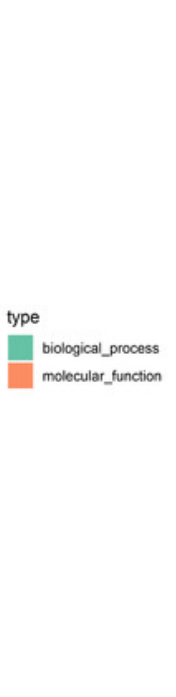

B

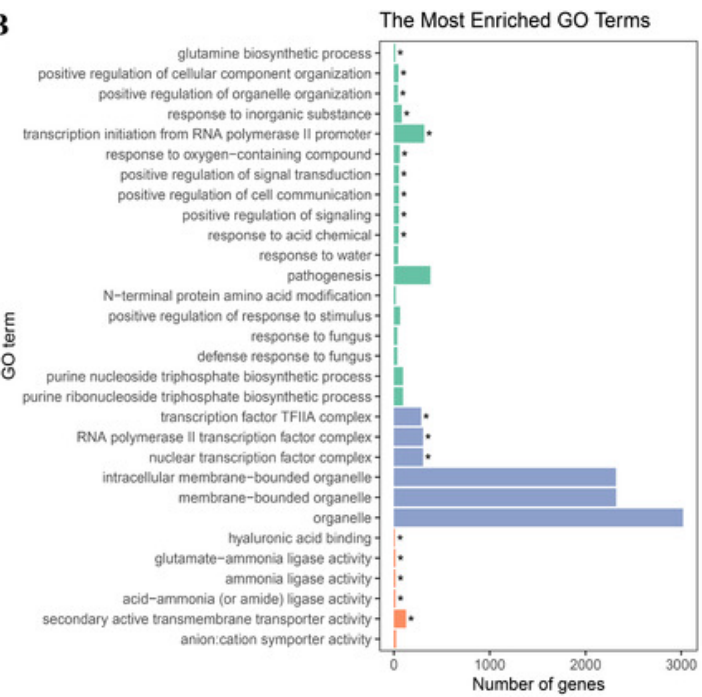

The Most Enriched GO Terms

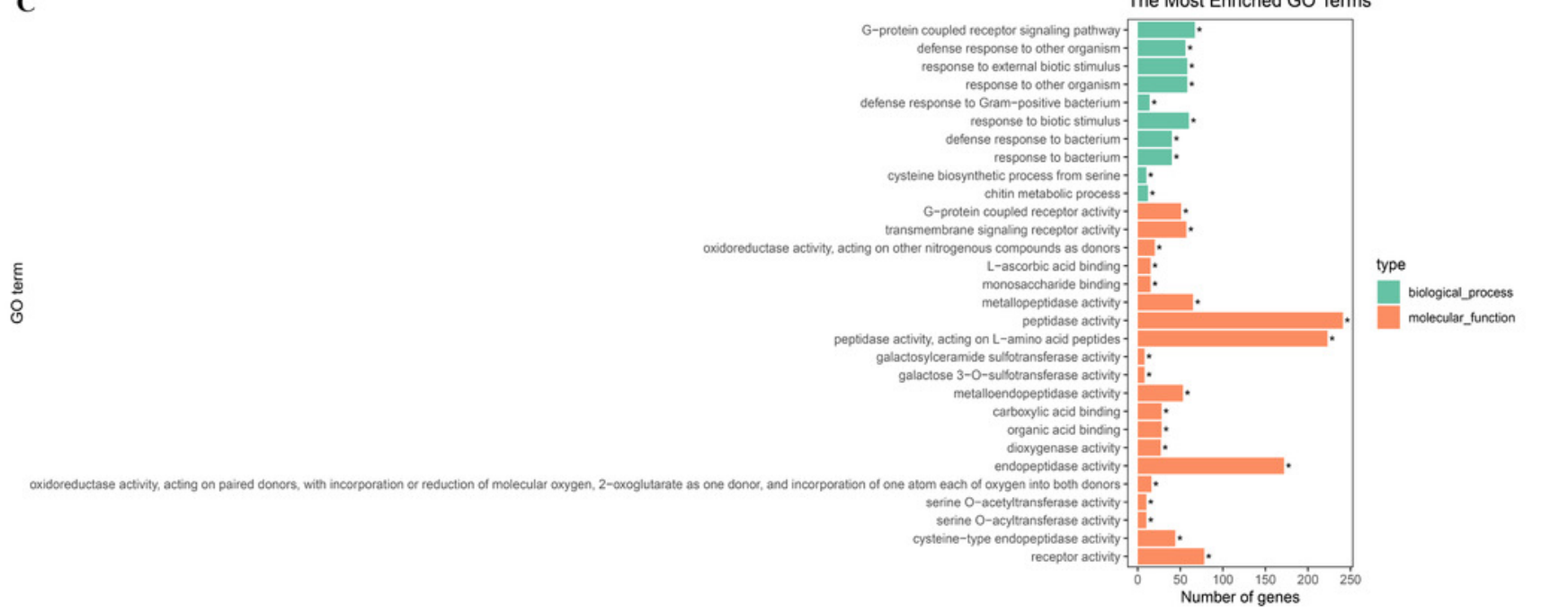

biological_grocess

celluluar_component

molecular_function 


\section{Figure 5}

Figure 5 KEGG enrichment of DEGs under salt stress.

(A) The top 20 most highly enriched KEGG pathways in 828 unigenes specifically differentially expressed under low salinity ( $0.1 \mathrm{M} \mathrm{NaCl}$ addition); (B) The top 20 most highly enriched KEGG pathways in 12,772 unigenes specifically differentially expressed under high salinity $(0.8 \mathrm{M}$ $\mathrm{NaCl}$ addition); and (C) the top 20 most highly enriched KEGG pathways in 2,802 unigenes differentially expressed under both high and low salinity. The names of the pathways and their enrichment factors are shown. The size of the dot indicates the number of genes in a given pathway, and the color of the dot indicates the value of $-\log 10$ (corrected $p$ value). 
$\mathbf{A}$

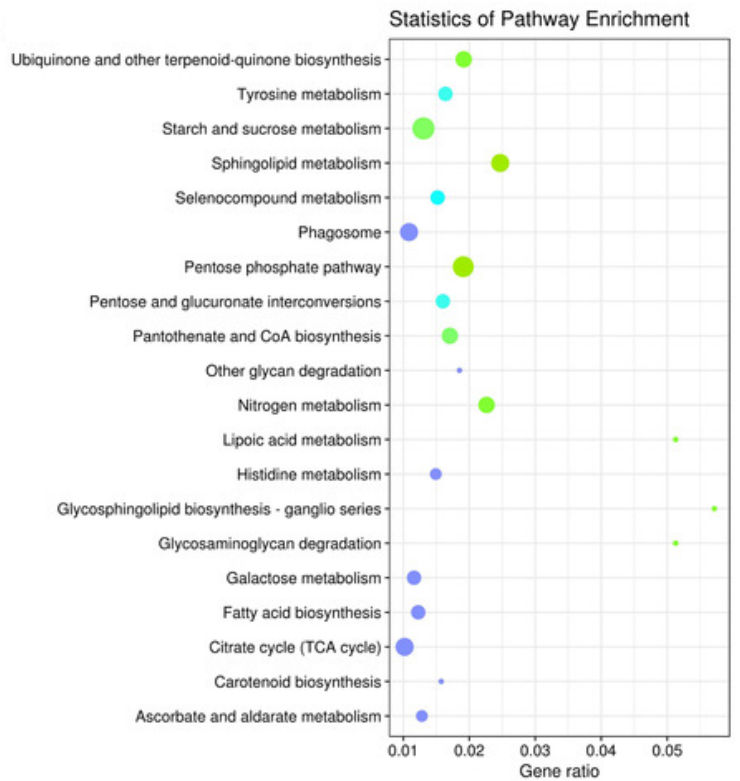

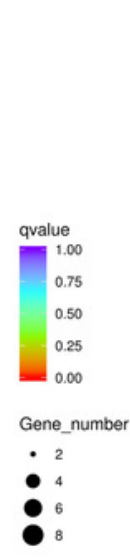

B

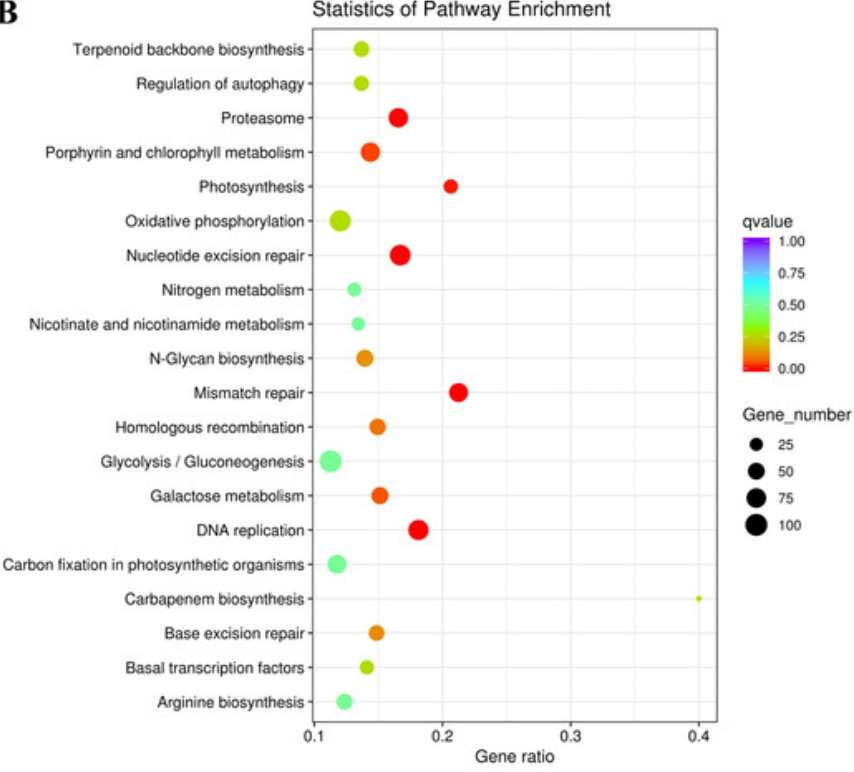

C

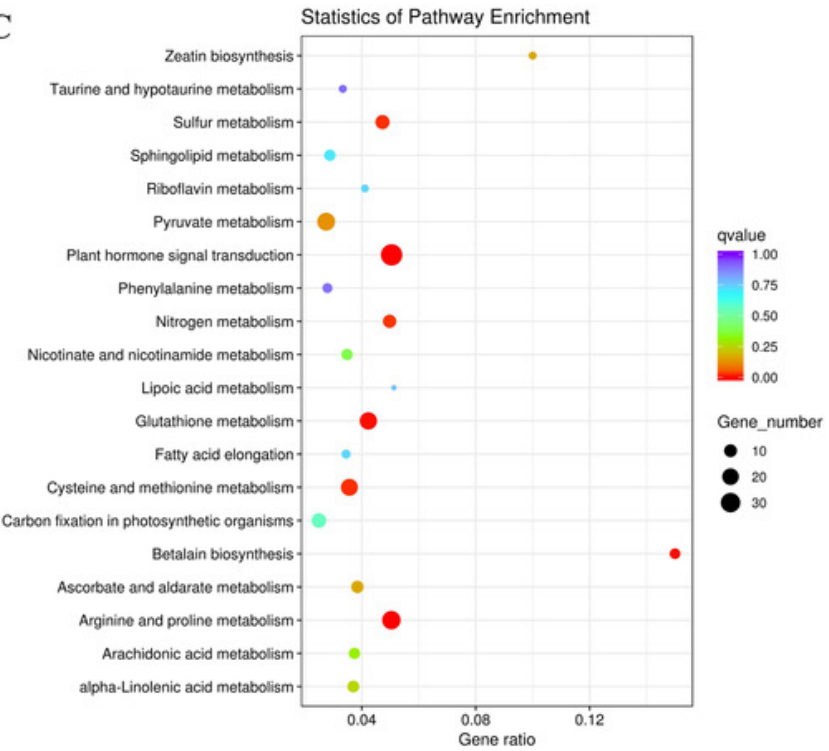




\section{Figure 6}

Figure 6 Enzymatic pathways of fatty acid and TAG biosynthesis and the expression of associated genes in response to two levels of salt treatment.

Arrows indicate enzymatic reactions, and red arrows indicate upregulation of the associated genes. Names of key lipid metabolism enzymes whose genes were up-regulated are given in red, names of enzymes whose genes were associated with lipid metabolism are given in blue, and names of enzymes whose genes were not differentially expressed are given in black. "-2" after an enzyme name indicates that its gene was differentially expressed under high salt stress (0.8 M NaCl addition). Enzymes without this designation were differentially expressed under both high and low (0.1 M NaCl addition) salt stress treatments. AMY, $\alpha$ amylase; HXK, hexokinase; PFK, 6-phosphofructokinase; DHAP, dihydroxyacetone phosphate; GAP, 3-phosphoglyceraldehyde; PYR, pyruvate; G3P, glycerol-3-phosphate; GPD, glycerol-3phosphate dehydrogenase; GK, glycerol kinase; ACC, acetyl-CoA; ACL, ATP citrate lyase; ME, malic enzyme; LACS, long-chain acyl CoA synthetase; MCAT, malonyl-CoA:acyl-carrier protein transacylase; SAD, stearoyl ACP desaturase; FAD, $\omega-3$ fatty acid desaturase; DGAT, diacylglycerol O-acyltransferase; TAG, triglyceride. Additional abbreviated gene names are defined in Figure 3. Gene expression levels are shown in Figure 7, Figure 8 and Table S10. 


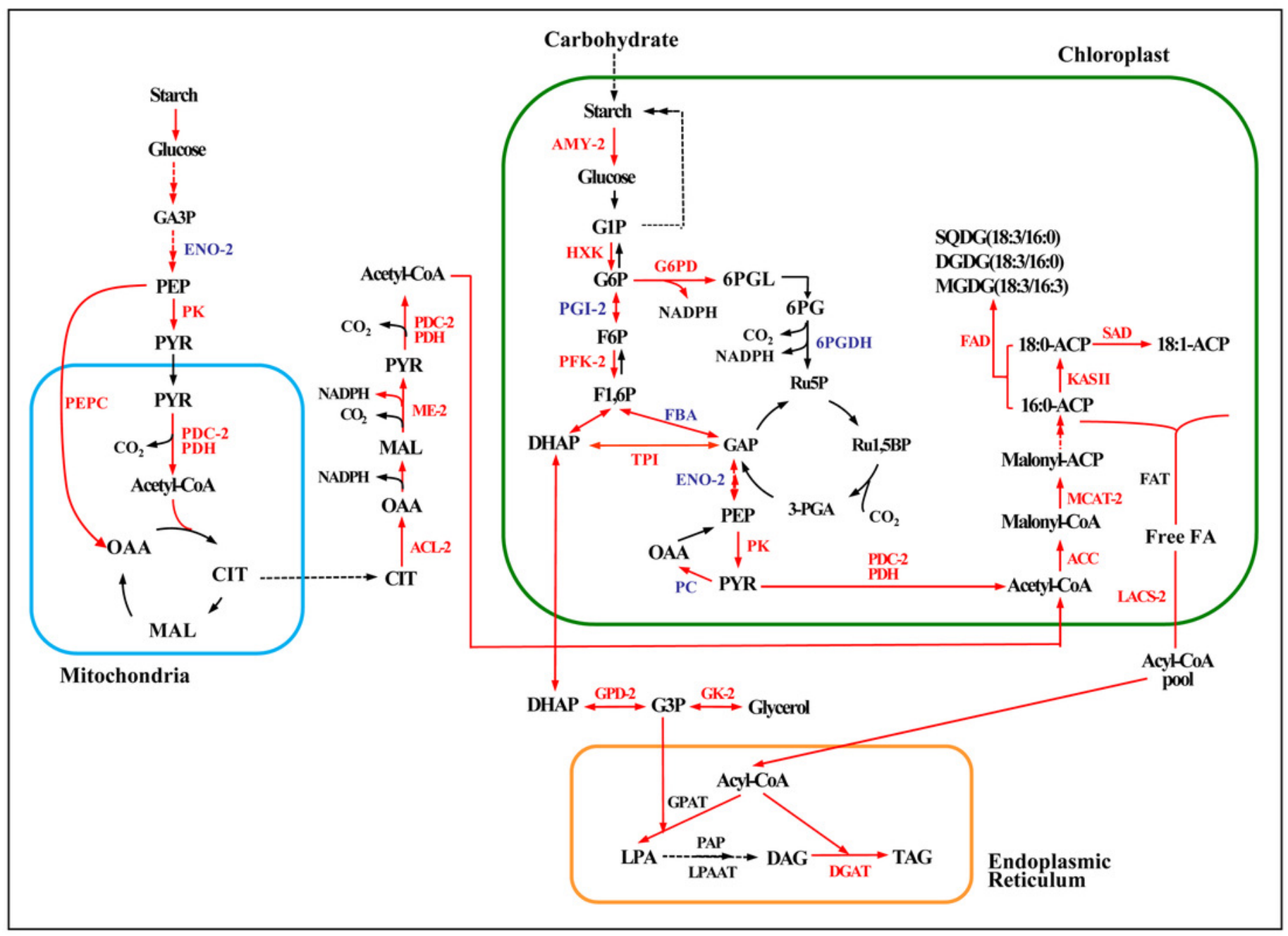




\section{Figure 7}

Figure 7 Expression of Chlorella sp. TLD6B genes involved in the de novo synthesis of fatty acids pathway under low $(0.1 \mathrm{M} \mathrm{NaCl}$ addition, $\mathrm{Nacl} 1)$ and high $(0.8 \mathrm{M} \mathrm{NaCl}$ addition, Nacl2) salt treatments.

(A) Expression of $\mathrm{BC} / \mathrm{BCCP} / \alpha-\mathrm{CT}$ genes; $\mathrm{BC}$, biotin carboxylase of ACCase; $\alpha-\mathrm{CT}$, carboxyltransferase $\alpha$-subunit; (B) Expression of MCTA gene; (C) Expression of KAS II genes; (D) Expression of FAD genes; (E) Expression of SAD genes. Gene IDs such as Cluster-31803.100315 are abbreviated as $\mathrm{Cl}-3.100315$. Additional abbreviated gene names are defined in Figures 3 and 6. 
A
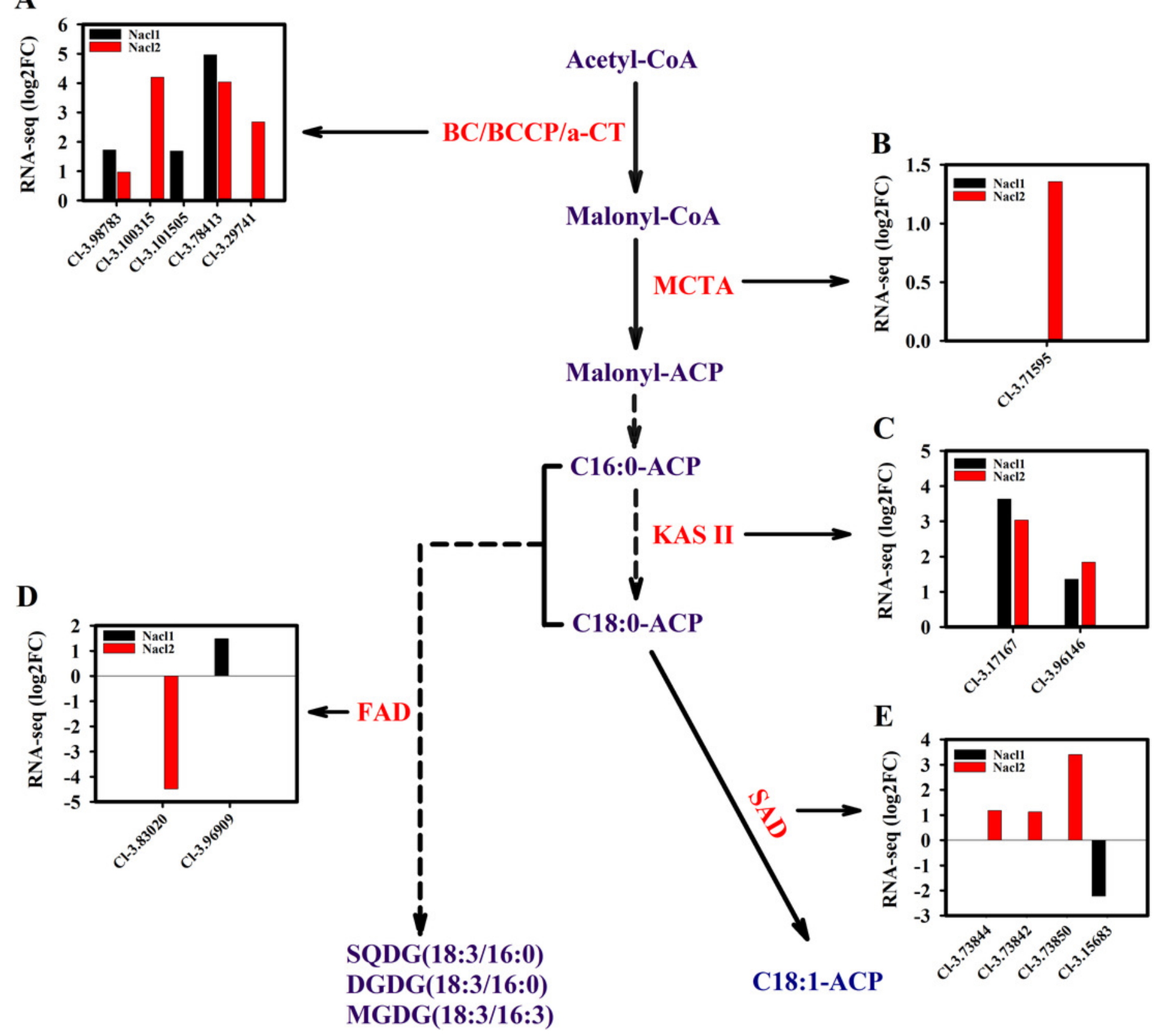

C

C16:0-ACP

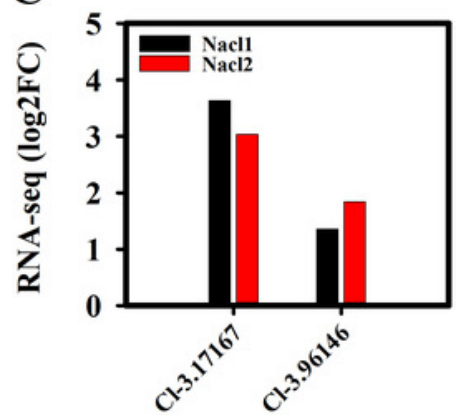

E
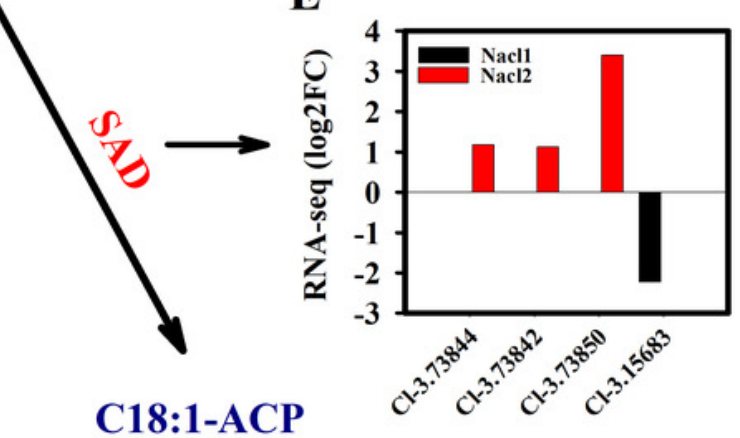

C18:1-ACP 
Figure 8

Figure 8 Expression of Chlorella sp. TLD6B genes involved in the TAG biosynthesis pathway under low (0.1 M NaCl addition, $\mathrm{Nacl}$ ) and high (0.8 M NaCl addition, Nacl2) salt treatments.

(A) Expression of TPI genes; (B) Expression of GPD genes; (C) Expression of GK gene; (D) Expression of DGAT genes. Gene IDs such as Cluster-31803.100315 are abbreviated as $\mathrm{Cl}-3.100315$. Additional abbreviated gene names are defined in Figures 3 and 6 .

A

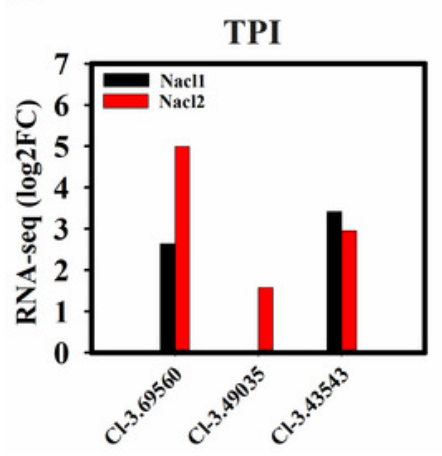

B

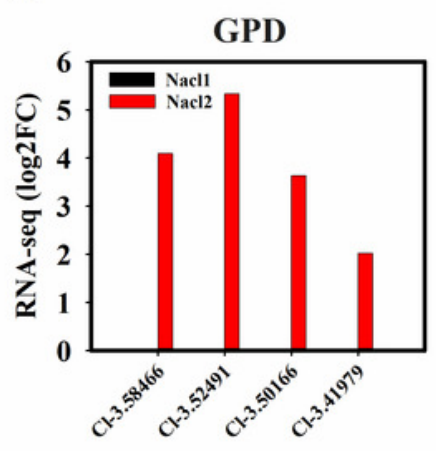

C

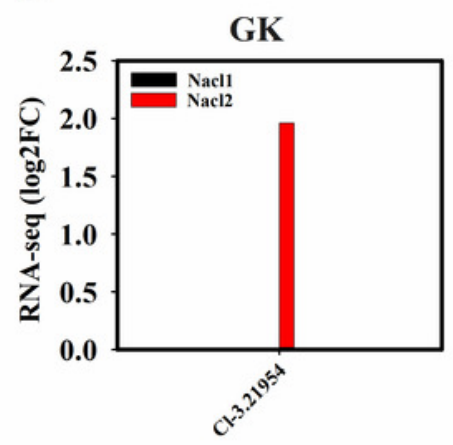

D

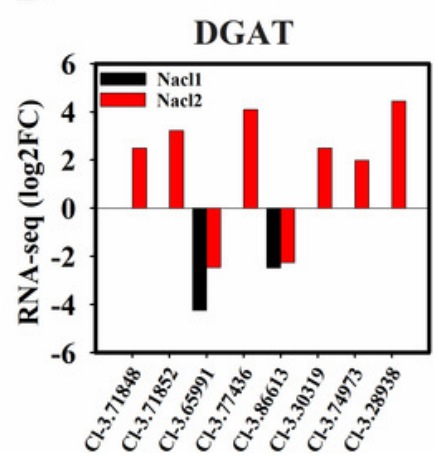




\section{Table 1 (on next page)}

Table 1 Correlation coefficients between biomass and physiological parameters under salt stress of different durations.

$* P<0.05$. B_6 indicates the biomass on day 6 , and so forth. 
1 Table 1 Correlation coefficients between biomass and physiological parameters under salt stress of different durations.

\begin{tabular}{|c|c|c|c|c|c|}
\hline Biomass under different stress durations & & $\mathrm{OD}_{680}$ & Carbohydrate concentration & Total lipid concentration & Adding $\mathrm{NaCl}$ concentration \\
\hline \multirow{2}{*}{ B_6 } & Pearson correlation & $0.822^{*}$ & $0.829^{*}$ & 0.607 & -0.894 \\
\hline & Significance (bilateral) & 0.045 & 0.041 & 0.201 & 0.016 \\
\hline \multirow{2}{*}{ B_12 } & Pearson correlation & $0.900^{*}$ & 0.787 & 0.578 & -0.736 \\
\hline & Significance (bilateral) & 0.014 & 0.063 & 0.229 & 0.096 \\
\hline \multirow{2}{*}{ B_18 } & Pearson correlation & $0.833^{*}$ & $0.905^{*}$ & 0.79 & -0.827 \\
\hline & Significance (bilateral) & 0.040 & 0.013 & 0.061 & 0.042 \\
\hline \multirow{2}{*}{ B_24 } & Pearson correlation & $0.904^{*}$ & $0.909^{*}$ & 0.62 & -0.935 \\
\hline & Significance (bilateral) & 0.013 & 0.012 & 0.189 & 0.006 \\
\hline
\end{tabular}

$2 * P<0.05$. B 6 indicates the biomass on day 6 , and so forth. 
Table 2 (on next page)

Table 2 Gene functional annotation. 
1 Table 2 Gene functional annotation.

\begin{tabular}{lll}
\hline & Number of unigenes & Percentage (\%) \\
\hline Annotated in NR & 111238 & 71.53 \\
Annotated in NT & 60439 & 38.86 \\
Annotated in KEGG & 47032 & 30.24 \\
Annotated in SwissProt & 84173 & 54.12 \\
Annotated in PFAM & 105026 & 67.53 \\
Annotated in GO & 109098 & 70.15 \\
Annotated in KOG & 51484 & 33.1 \\
Annotated in all databases & 23373 & 15.03 \\
Annotated in at least one database & 131623 & 84.64 \\
Total unigenes & 155503 & 100 \\
\hline
\end{tabular}

